\title{
- A within-host infection model to explore tolerance and resistance
}

\author{
Pierre D. M. Lafont*1,2, Christine Lauzeral*1, Nathalie Parthuisot ${ }^{1}$, \\ Christian Faucher ${ }^{1}$, David Duneau ${ }^{\dagger \ddagger 1,3}$, and Jean-Baptiste Ferdy ${ }^{\ddagger 1}$ \\ ${ }^{1}$ Évolution et Diversité Biologique UMR 5174 CNRS-UPS, Université Paul Sabatier, F-31062 \\ Toulouse cedex 9, France \\ ${ }^{2}$ Institute of Evolutionary Biology, School of Biological Sciences, University of Edinburgh, \\ Edinburgh EH9 3FL, United Kingdom \\ ${ }^{3}$ Instituto Gulbenkian de Ciência, Oeiras, Portugal
}

\begin{abstract}
How are some individuals surviving infections while others die? The answer lies in how infected individuals invest into controlling pathogen proliferation and mitigating damage, two strategies respectively called resistance and disease tolerance. Pathogen within-host dynamics (WHD), influenced by resistance, and its connection to host survival, determined by tolerance, decide the infection outcome. To grasp these intricate effects of resistance and tolerance, we used a deterministic theoretical model where pathogens interact with the immune system of a host. The model describes the positive and negative regulation of the immune response, consider the way damage accumulate during the infection and predicts WHD. When chronic, infections stabilize at a Set-Point Pathogen Load (SPPL). Our model predicts that this situation can be transient, the SPPL being then a predictor of life span which depends on initial condition (e.g. inoculum). When stable, the SPPL is rather diagnostic of non lethal chronic infections. In lethal infections, hosts die at a Pathogen Load Upon Death (PLUD) which is almost independent from the initial conditions. As the SPPL, the PLUD is affected by both resistance and tolerance but we demonstrate that it can be used in conjunction with mortality measurement to distinguish the effect of disease tolerance from that of resistance. We validate empirically this new approach, using Drosophila melanogaster and the pathogen Providencia rettgeri.
\end{abstract}

\section{Introduction}

Infectious diseases produce a vast array of symptoms, some leading to death whereas others are easily overcome. This heterogeneity of outcomes reflects in part the diversity of pathogens, but even benign pathogens can sometimes kill while the most deadly ones seldom cause 100\% mortality (e.g. even in Ebola infections, $40 \%$ of infected people survive, Shultz et al., 2016). Predicting who is at greater risk of dying from an infection is central in medicine,

${ }^{*}$ These authors contributed equally to this work.

†david.duneau@gmail.com

¥These authors also contributed equally to this work.

§jean-baptiste.ferdy@univ-tlse3.fr 
because accurate predictions can help tailoring public health efforts. A common approach to this problem relies on detecting statistical associations between patients' conditions and fatality rates (e.g. Barda et al., 2020, in the case of COVID-19). Although essential and efficient, this approach is not meant to understand the ultimate causes of death.

What actually makes an infected patient succumb is that it did not control pathogen proliferation and could not sustain the damage imposed by the infection (Colaço et al., 2020; Jackson et al., 2014; Soares et al., 2017). Overall, the hosts can therefore survive an infection by a combination of strategies which involve resisting to pathogens by producing immune defense and tolerating the consequences of infection (in animals (Ayres \& Schneider, 2008; Råberg et al., 2007; Read et al., 2008), but also in plants (Kover \& Schaal, 2002)). Hosts which engage in the second type of strategy either sustain a high level of damage (i.e. disease tolerance stricto sensu) or repair them efficiently to maintain homeostasis (i.e. resilience sensu Ferrandon (2013)). Forecasting the outcome of an infection for one individual patient therefore requires that we quantify the relative investment in each of these strategies.

In principle, separating the effects of disease resistance from those of disease tolerance should be relatively easy, as resistance directly impacts the Within Host Dynamics (WHD) of pathogens while tolerance affects survival but should have no effect on WHD. The task is in fact challenging because both resistance and tolerance have indirect effects which intermingle. For example, immune defense are a double-edged sword which can save the host's life but sometimes cause pathologies (Petkau et al., 2017) and reduce lifespan (Y. Lin et al., 2018). An increase in resistance could therefore come with an apparent decrease in disease tolerance. Reciprocally, disease tolerance and the damage mitigation it relies on could allow a host to sustain higher levels of defense, and indirectly increase its resistance to disease. These intricate effects, added to the fact that some regulatory genes affect both resistance and tolerance, led some authors to consider that they should be seen as two finely co-regulated aspects of the immune response to infection (Martins et al., 2019; Pucillo \& Vitale, 2020). Studying experimentally the WHD and its connection to survival is probably still the only way to understand the mechanisms which underlie resistance and tolerance, but we advocate that theoretical studies are needed to unravel their effects and provide testable predictions.

We present here an effort to disentangle the action of disease tolerance and resistance using a mathematical model of WHD. Our model aims at being general, while taking advantage of recent empirical descriptions of WHD in Drosophila melanogaster (Duneau et al., 2017a). Invertebrates have proven to be good alternative experimental models to understand the relation between WHD and infection outcomes. First, they provide the advantage that total pathogen load can be quantified on large numbers of animals, regardless of the pathogen tropism, so that the WHD can be studied on a wide range of disease types (Duneau et al., 2017a). Second, they share with mammals a large part of the mechanisms underlying their innate immune system, as shown in Drosophila melanogaster (Buchon et al., 2014). Our purpose was two-fold: first, we aimed at designing a model which could reproduce the different situations documented by Duneau et al. (2017a) and in other studies; second we used it to explore how experimental proxies of resistance and disease tolerance actually connect to infection parameters. Figure 1 presents an example of WHD in Drosophila which illustrates one of the behaviour we wanted our model to be able to reproduce, and defines the Pathogen Load Upon Death (PLUD) and the Set Point Pathogen Load (SPPL, which we propose as a generalization of SPVL and SPBL to pathogens other than viruses and bacteria), the two 


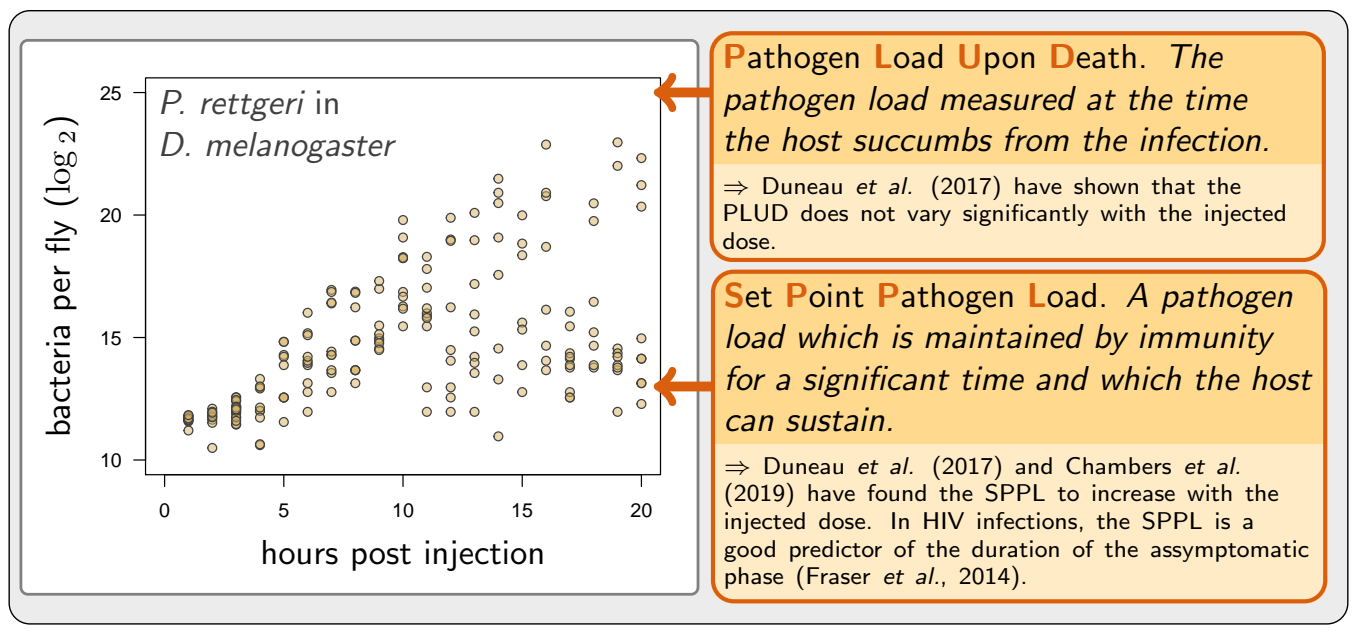

Figure 1: Adapted from Duneau et al. (2017a). Each point represents the bacterial load estimated from a single killed male fly (Drosophila melanogaster) injected with a suspension of the bacterium Providencia rettgeri containing ca. 2,000 bacterial cells. Twenty hours after injection, some flies maintain a moderate load and will live on for several days, while others have reached high loads and will die rapidly.

quantities which we have investigated most. Finally, we used our model to design methods which could help to experimentally distinguish the effects of disease tolerance from that of resistance.

We found that only models with complex, non-linear regulations of immunity can satisfactorily reproduce infections which range from chronic to acute and from benign to lethal. We further demonstrated that the model adequately predicts both the existence and the properties of the SPPL if defense production is assumed to decrease with accumulating damage. We show that the SPPL can then be transient and does, as documented in HIV infections, predict the host's lifespan. We further demonstrated that SPPLs with entirely different properties can be measured in other situations where the pathogen stably associates to the host, causing little damage. Our model also predicts that the host dies at a PLUD which is virtually independent from inoculum size (as observed by Duneau et al., 2017a), and should therefore reflect the genetic characteristics of interacting pathogens and hosts. We finally propose that the PLUD could be used in combination with a mortality measurement (such as the Hazard Ratio) to experimentally distinguish the effects of disease tolerance from that of resistance. We validated this theory using experimental infections of Drosophila melanogaster by the pathogenic bacterium Providencia rettgeri.

\section{A Lotka-Volterra model of host-pathogen interac- tions}

One purpose of our work was to develop a mathematical model able to reproduce the great variety of WHD documented to date. Our model aims at being general, but we used the recent empirical work of Duneau et al. (2017a) on Drosophila melanogaster to facilitate 
the explanations and the interpretations. This previous work documents chronic and acute diseases, some being benign while others are lethal. But most importantly it also depicts situations where an infection initiated in carefully controlled conditions can bifurcate, with some of the hosts developing a form of chronic infection when others die within a few days (as illustrated in fig. 1).

Such bifurcations in dynamical systems are most often by-products of bistability. The few available examples of bistable WHD models in literature are modifications of the LotkaVolterra (LV) model (King et al., 2009; Pujol et al., 2009; Souto-Maior et al., 2018). Recently, van Leeuwen et al. (2019) demonstrated that a LV model which explicitly describes the amount of resource diverted from the host by intestinal pathogens can be bistable. Ellner et al. (2021) showed that situations where pathogens actively destroy defenses also create bistability in disease outcome. These two models have in common the fact that pathogen proliferation negatively impacts immune defense. Louie et al. (2016) proposed a LV model with an explicit description of how damage accumulates over the course of the infection, but they did not investigate the bistability of their model. Most importantly, all these models allow bifurcations only between clearance and chronic equilibrium. Ellner et al. (2021) may be the exception but they considered the infection as being almost cleared, as they found that immunity stabilizes pathogen loads at very low levels. In an older work, Mayer et al. (1995) demonstrated that non-linear functional responses for both pathogen dynamics and host defense regulation could yield bifurcations between chronic infections with high and low equilibrium pathogen load. Following these ideas, we considered a LV model where pathogens inflict damage to their host, so that our model can predict both the pathogen dynamics and the survival chances of the host, and where defense are modulated non linearly according to pathogen load and to damage.

Let $x$ be the pathogen load (e.g. bacteria), $y$ the level of defense (e.g. anti-microbial peptides) produced by the host immune system and $z$ the damage caused by the infection (see fig. 2A). Whether the host suffers from severe infection, chronic yet mild infection, or clears pathogens, depends on its genetically determined capacity to fight or tolerate pathogens, but also on the inoculum size and on the host physiological state at the start of the infection. In our model, the inoculum size is the initial value of the pathogen load $\left(x_{0}\right)$. The physiological state of the host at the start of the infection is described by the initial level of defense $\left(y_{0}\right)$ and of damage $\left(z_{0}\right)$. The initial level of defense can be set by constitutive immunity, when a healthy host is at its homeostatic state (i.e. $y_{0}=\tilde{y}_{h}$ ). It can also be lowered, when the host is weakened by environmental challenges. Similarly, before the infection has started, the host suffers from an initial level of damage which origin is not the studied infection. This level is $\tilde{z}_{h}$ when the host is at its homeostatic state, but can be increased in case of environmental challenges (e.g. infection starting from a wound).

The genetically determined capacity of the host to fight an infection is characterized by a set of parameters (described in fig. 2A) which are fixed for a given host and pathogen. These parameters govern the dynamics of $(x, y, z)$ over time $t$, as described by the following 
differential equations:

$$
\left\{\begin{array}{l}
\frac{d x}{d t}=x(1-x)-\delta x y \\
\frac{d y}{d t}=F(x) G(y, z)-\varphi y \\
\frac{d z}{d t}=\omega x+\eta y-\xi z .
\end{array}\right.
$$

For the sake of simplicity, the first equation of system (1) is written dimensionless, $x$ being a fraction of the pathogen's carrying capacity within the host and time units corresponding to the pathogen's generation time. The equation governing the dynamics of pathogens is that of the evolution of a prey population in a classic Lotka-Volterra model (Lotka et al., 1923; Volterra, 1928). The pathogens are destroyed by the defense at a rate $\delta y$, with $\delta$ representing the efficacy of immune molecules or cells at killing pathogens.

We considered that damage should increase with pathogen load, but that it might also increase with the level of defense that are produced. We assumed that damage are caused by pathogens at a rate $\omega$ and by defense at a rate $\eta$. We further reasoned that damage are repaired at a constant rate $\xi$. It is now well established that repairing mechanisms are in fact modulated during the course of an infection (Martins et al., 2019; Pucillo \& Vitale, 2020). For the sake of simplicity, and because the details on how this regulation responds to damage and defense production are still unknown, we purposely neglected this modulation. Finally, we postulated that the host cannot sustain infinite damage, so that it will die if $z$ exceeds the threshold value $z_{d}$.

We assumed that the time variation in the level of defense is set by the balance between the production of new molecules or cells $(F(x) G(y, z))$ and the spontaneous decay of already produced ones ( $\varphi y$, with defense persisting longer in the host when $\varphi$ is decreased). The functions $F$ and $G$ describe the modulation of defense production, with $F$ modeling how production is activated upon pathogen detection, and $G$ describing negative regulation. Following Mayer et al. (1995), we wrote both $F$ and $G$ as non-linear functional responses and investigated how the shapes of these two functions influence the outcome of the infection.

The function $F$ is given by

$$
F(x)=\gamma+\alpha \frac{x^{u}}{1+\beta x^{v}}
$$

We posited that $F$ must be an increasing function of pathogen load ( $u \geq v$ and $\beta>0$ ). In the absence of pathogens and with no negative regulation, the production of defense equals $\gamma$. The parameter $\gamma$ therefore sets the maximum constitutive production of defense. Upon infection, $F$ increases with the pathogen load, and reaches its maximum feasible value when $x$ reaches carrying capacity (with then $F(1)=\gamma+\alpha /(1+\beta)$ ). Figure $2 \mathrm{~B}$ illustrates that higher values of $\alpha$, i.e. more sensitive defense activation, make this increase steeper. Increasing $u$ above $v$ makes $F$ sigmoidal, which reproduces cases where the production of defense is barely activated unless pathogen load becomes significant (as fig. 2B illustrates). Overall, the parametrization of $F$ allowed us to vary the constitutive level of defense expression, the speed at which defense are activated upon infection, and the load at which pathogens are detected. 


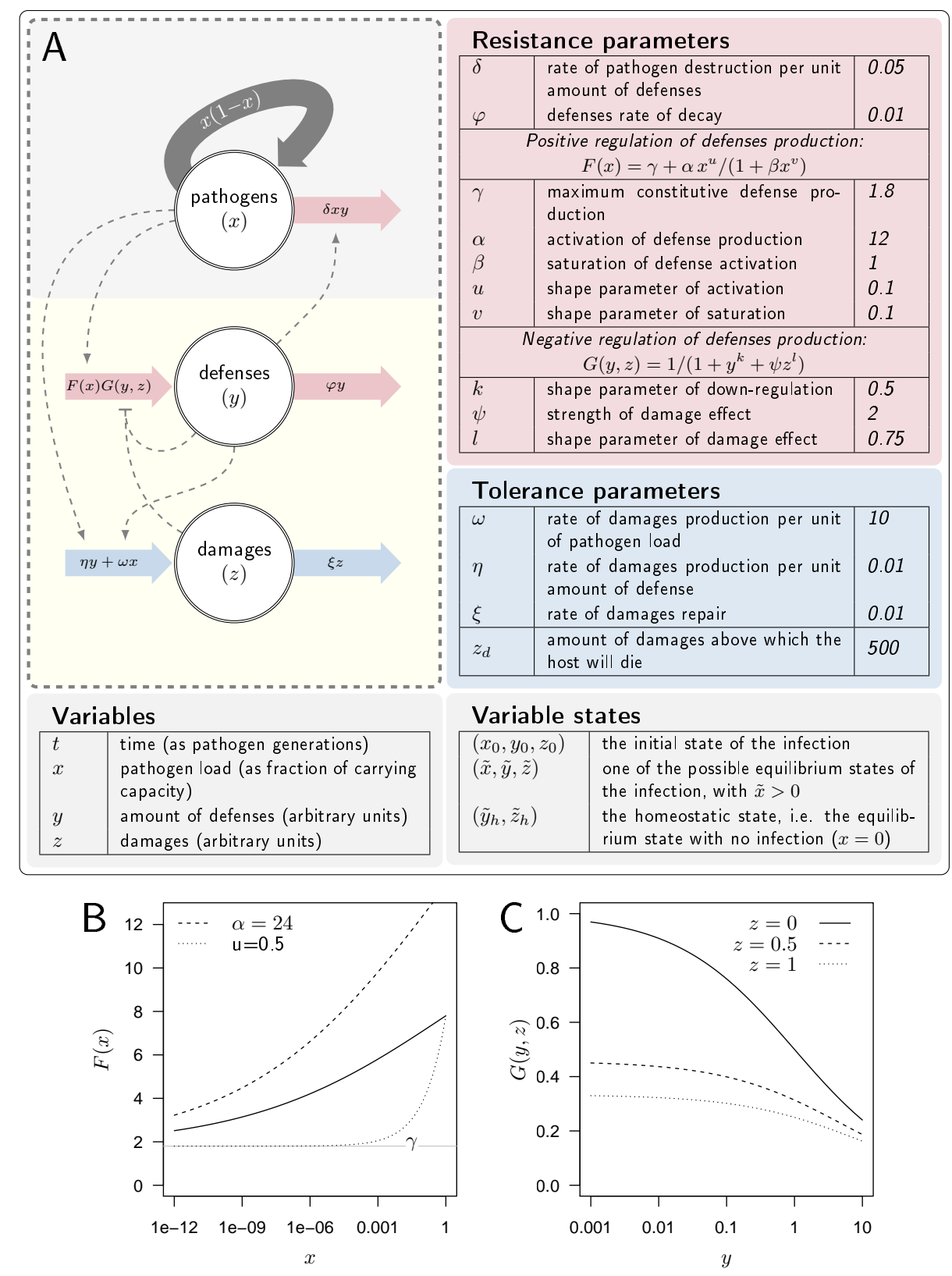

Figure 2: A. A description of the model. Large arrows indicate fluxes which make pathogen load $(x)$, level of defense $(y)$ and damage $(z)$ vary over time. Dashed arrows indicate how each variable influences, negatively (flat arrowhead) or positively, these fluxes. Tables list parameters and their default values, separating those which determine the production or efficacy of defense (resistance parameters) from those which determine the production and the repair of damage (tolerance parameters). B. The activation of defense as described by the functional response $F(x)$. $F$ gives the level of defense production that is reached in the absence of negative regulation. It increases with pathogen load $x, F(0)=\gamma$ (the horizontal gray line) being the maximum constitutive defense production, reached when the host is not infected. $\alpha$ controls how fast defense expression increases with load (dashed curve: $\alpha=24$ instead of 12 for the plain curve). Increasing $u$, (dotted curve: $u=0.75$ instead of 0.5 ) makes $F$ increase slower when pathogen load is low. C. The negative regulation of defense production as described by the functional response $G(y, z)$. Down-regulation of defense production increases with both defense $(y)$ and damage $(z)$ levels. Note however that $\psi=0$ or $l=0$ would make $G$ independent of damage level. 
The function $G$ corresponds to the negative regulation of the production of defense, with

$$
G(y, z)=\frac{1}{1+y^{k}+\psi z^{l}} .
$$

Here, we considered that the regulation of the production of defense must incorporate a negative effect of immune defense $(y)$ on defense production itself. This was motivated by the negative feedback loops that regulate immune response in mammals and insects. In Drosophila, for example, the PGRP-LB gene, which has mammalian orthologs, is activated by the immune deficiency (Imd) pathway, one of the signaling cascades which regulates the expression of most antimicrobial peptides. But PGRP-LB also down-regulates this same Imd pathway, and thus provides a clear example of negative regulation of immune response (Kleino \& Silverman, 2014). The negative effect of the damage $(z)$ on defense production (see fig. $2 \mathrm{C}$ ) is motivated by the idea that the damage caused by an infection disrupts immune system homeostasis by altering host health condition (Stoecklein et al., 2012). This may be because the energy spent in repairing damage is no longer available to produce defense. It may also be that the tissues producing defense are themselves damaged by the infection, hence impairing the production of immune molecules or cells. The weight of these effects on the production of defense is controlled by the parameters $\psi$ and $l$.

As should be clear from equations (2) and (3), we purposely made $F$ and $G$ versatile so that our model can reproduce various types of host-pathogen interactions. This came at the cost of a large number of parameters. Figure 2 represents the model, lists its parameters and gives the parameter values we have used in our computations, unless otherwise specified. Parameters can be separated in two lists, with disease tolerance parameters that control damage production or repair on one side, and resistance parameters, which control the efficacy and level of immune defense on the other (see fig. 2). This clear-cut distinction, though, ignores potential indirect effects. For example, increasing the host capacity to repair damage (i.e. increasing $\xi$ ) makes it more tolerant to the infection. But damage hinders defense production, so that increasing $\xi$ indirectly allows the host to produce more defense, hence making it more resistant. One of our aims was therefore to describe how the combined direct and indirect effects of each parameter impact resistance and disease tolerance to infection.

Finally, due to the complexity of functions $F$ and $G$, the equilibria of our model are intractable. We therefore relied on numerical methods to compute both the homeostatic state in the absence of pathogens $\left(\tilde{y}_{h}, \tilde{z}_{h}\right)$ and other equilibria $(\tilde{x}, \tilde{y}, \tilde{z})$ with pathogens present. This, as most of the numerical analysis detailed here, has been performed using the GSL library (Galassi et al., 2009) and functions provided in R (R Core Team, 2018). We were nevertheless able to derive the stability conditions for some of the equilibria (see Supplementary Material S1) and the relation between equilibrium load and parameters (see Supplementary Material S2).

\section{The different possible outcomes of an infection}

The entanglement between pathogen proliferation, defense and damage production is too complex to predict the outcome of an infection from their separate effects. But numerically 

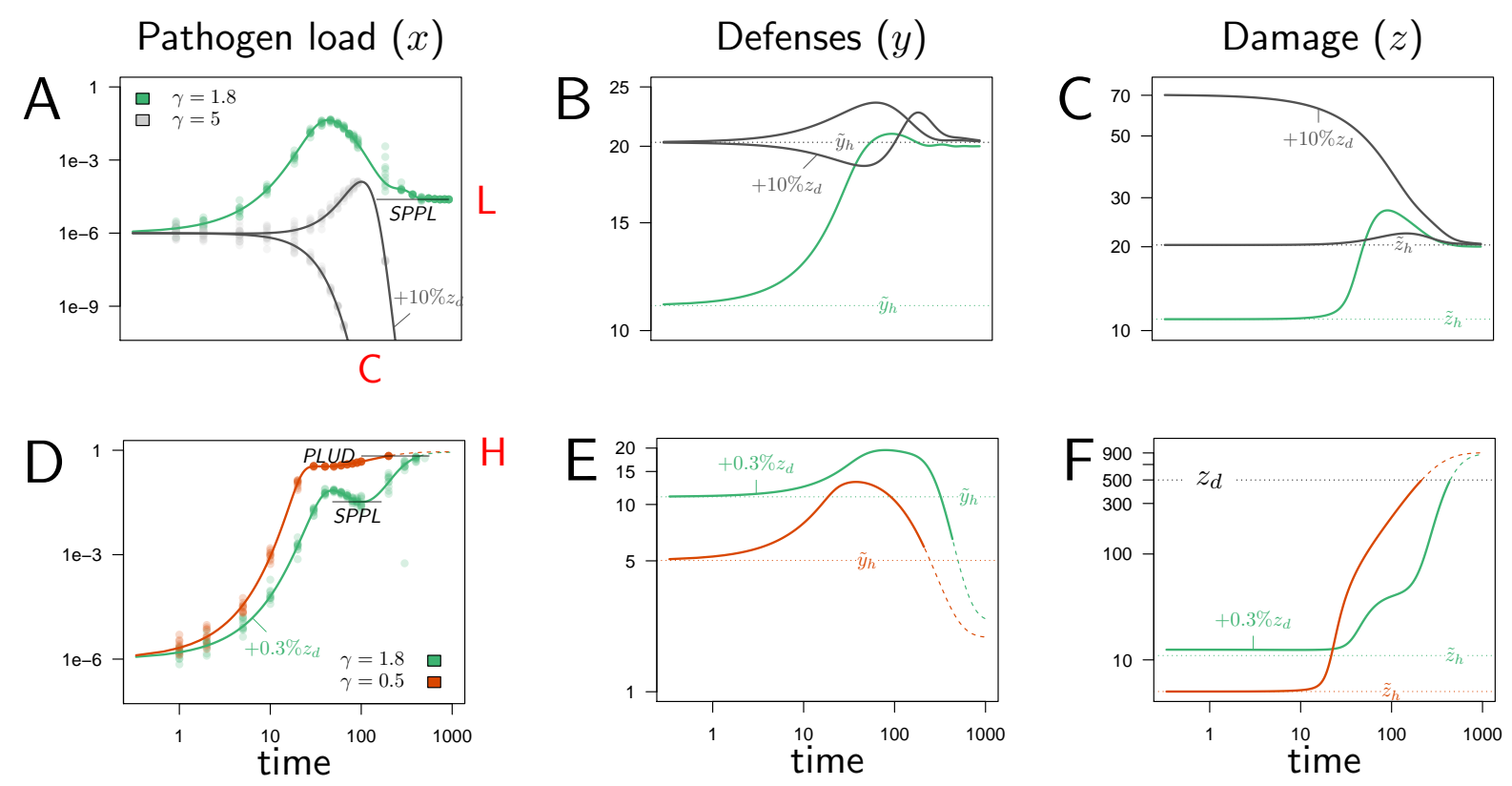

Figure 3: Illustrative cases of infection dynamics. Each column corresponds to one of the three variables of the model ( $x$, pathogen load; $y$, defense; $z$, damage). In all cases, the initial pathogen load $\left(x_{0}\right)$ is $10^{-6}$ and, unless otherwise specified, $y_{0}$ and $z_{0}$ are set at their homeostatic state $\tilde{y}_{h}$ and $\tilde{z}_{h}$ (indicated by horizontal dashed line). Red letters indicate the outcome of the infection: C, clearance; L, low pathogen load; H, high pathogen load. Dots corresponds to results of stochastic simulations where the initial pathogen load is randomly drawn from a log-normal distribution with average in $\log \left(x_{0}\right)$ set to $10^{-6}$ and variance set to 0.15 . A-C illustrate cases where the immune defense eventually control the infection, either by clearing pathogens (gray curves, $\gamma=5$ ) or by stabilizing the infection, making it chronic (green curves, $\gamma=1.8$ ). With $\gamma=5$, the constitutive expression of defense is strong so that the homeostatic level of defense is very high. The level of defense slightly increases upon infection (which leads to a slight increase in damage, as defense induce damage when $\eta>0$ ) and returns to the homeostatic state once pathogens have been cleared. With $\gamma=1.8$ (green curve), the initial level of defense is much lower and the infection stabilizes at a Set Point Pathogen Load (SPPL, see fig. 1). Defense and damage peaks following infection and, because pathogen are not cleared, they eventually stabilize at a level above the homeostatic state. D-F illustrate cases where the immune response does not suffice to control the infection (green curves, $\gamma=1.8$, red curves, $\gamma=0.5$ ). In both cases, the peak in defense production that follows infection does not stop pathogens from proliferating. This, combined to the side effects of defense, provokes a sharp increase in damage which eventually kills the host, when $z$ exceeds the level the host can sustain $\left(z>z_{d}\right)$. The accumulation of damage hinders defense production (because $\psi>0$ and $l>0$ ) which provokes a sharp drop of defense and helps explaining why these infections are lethal. The two hosts die at very similar Pathogen Load Upon Death (PLUD, see fig. 1) although at different times. When $\gamma=1.8$ (green curve), the immune response is strong enough to curb pathogen proliferation. This maintains the population of pathogens at a SPPL, which differs from that of the green curve in figures A-C because it is transient. These two green curves have been obtained with identical parameters; they only differ in the initial level of damage $z_{0}$ (which,in $\mathrm{D}-\mathrm{F}$, is increased by $3 \%$ of $z_{d}$ ). Their differences therefore demonstrate that the model can be bistable: with fixed parameters, the outcome of an infection might depend on initial conditions. These simulations can be reproduced using a dedicated $\mathrm{R}$ Shiny web application (Chang et al., 2021), where parameters values can be changed to explore their impact on the dynamics (see https://plafont.shinyapps.io/WHD_app/). 
integrating equations (1) allowed us to simulate infections, and thus to investigate how variations in parameters (e.g. the maximum level of constitutive production of defense, $\gamma$ ) or initial variable values (e.g. the initial level of damage, $z_{0}$ ) impact WHD and eventually determine the survival of the host.

By presenting a few chosen simulations, we first showed that our model can reproduce situations where the host succeeds in controlling the infection (fig. 3A-C), either by clearing pathogens $(\gamma=5)$ or by maintaining pathogen load, and therefore damage, at a tolerable level $(\gamma=1.8)$. In the latter case, we can consider that the population of pathogens eventually reaches a Set Point Pathogen Load (SPPL, as defined in fig. 1) and will stay there for the whole host's life. In the three simulations of figures 3A-C, the level of defense was initially set at its homeostatic state, the immune state of a healthy host $\left(\tilde{y}_{h}\right)$. Upon infection, it increased and either returned to its initial level, when the infection is cleared, or stabilized to an intermediate level due to the remaining controlled pathogen population (fig. $3 \mathrm{~B}, \gamma=1.8$ ).

Hosts are expected to be at a greater risk of infection when external challenges disrupt their homeostatic state. A host may be, for example, infected through a wound; if this wound causes damage which in turn hampers the expression of immune defense, the infection is facilitated (Stoecklein et al., 2012). This has been illustrated in Chambers et al. (2014), where the type and the addition of aseptic injury to Drosophila melanogaster prior to infection increased mortality to Providencia rettgeri compared to infections without wound. We reproduced this situation by setting $z_{0}$ above the homeostatic level of damage $\tilde{z}_{h}$. In this case, defense first decreased under the effect of damage, and the immune response occurs with a delay (fig. $3 \mathrm{~B}$, gray curve with $\gamma=5$ and $z_{0}=\tilde{z}_{h}+10 \% z_{d}$ ). This allows pathogens to proliferate during early infection, but the host nevertheless managed to clear the infection and we demonstrated (see Supplementary Material S1.1) that this is because the strong constitutive production of defense $(\gamma=5)$ in this simulation guaranties a high homeostatic level of defense. More generally, we proved that clearance is possible if and only if $\gamma \geq \gamma_{c}$, with

$$
\gamma_{c}=\frac{\varphi / \delta}{G(1 / \delta, \eta /(\xi \delta))} .
$$

Complete healing is thus possible only when a sufficient level of defense is present at the homeostatic state, before the infection starts. As equations (4) demonstrates, this level decreases when defense are more efficient ( $\delta$ increases) or persist longer $(\varphi$ decreases $)$ and increases with down-regulation of defense production.

The wound did not change the outcome of infection in hosts with strong constitutive immunity. It did in hosts with $\gamma=1.8$, which survived infection when not wounded (green curve in fig. 3A) but died from it when wounded prior to infection $\left(z_{0}=\tilde{z}_{h}+3 \% z_{d}\right.$ in the green curves of fig. 3D). The initial immune handicap caused by the wound indeed facilitated infection, so that pathogen load reaches extreme values which eventually killed the host. Environmental challenges other than wounds might have a direct negative impact on defense production, which we could reproduce in our model by setting $y_{0}$ below $\tilde{y}_{h}$. This type of challenge could also facilitate infections by opportunistic microorganisms, with trajectories similar to the green curve of figure 3D-F. As expected, hosts with weak constitutive defense can die from infection even when not wounded $(\gamma=0.5$, red curves fig. 3D-F). In the two lethal infections presented in figures 3D-F, defense decreased as damage rapidly accumulated during the final phase of the disease (see fig. $3 \mathrm{E}$ ), because $\psi>0$ makes damage hinder 
defense production. In both cases, damage eventually exceeded the maximum level the host can sustain $\left(z_{d}\right)$, and the host died from the infection (see fig. $3 \mathrm{~F}$ ).

Although identical in their final outcome, the two simulations of figures 3D-F differ in the time the infection took to kill the host. When constitutive immunity is weak (red curve, $\gamma=$ 0.5), pathogens reached high loads before defense managed to slow down their proliferation. The hosts therefore succumbed rapidly. When constitutive immunity is stronger but the host is wounded (green curve, $\gamma=1.8$ ), the host went through a phase where pathogen proliferation was under temporary control, and infection transiently stabilized at a SPPL. This SPPL differs fundamentally from that of figure 3A-C, because slowly accumulating damage ended up bringing defense below the level which permits efficient control of pathogen proliferation. This SPPL was therefore transient, while that of figure 3A-C was stable.

\section{Bistability can make the SPPL transient}

The host depicted by the green curves of figures 3A-C suffered from a chronic but benign infection; that of the green curves of figures 3D-F died from a severe infection. Still these two hosts and their respective pathogens can be considered as genetically identical, as the two simulations were ran with the very same parameter values. The only difference between them was that the host of figures 3D-F has been wounded before being infected. This result demonstrates that our model can be bistable: for a fixed set of parameters, the outcome of an infection can depend on initial conditions.

We were not able to determine sufficient conditions which would guarantee bistability, but we demonstrated that as long as the production of defense (represented by $F$, fig. 2B) increases with $x$, bistability cannot occur unless $\psi>0$ (Supplementary Material S1.2). Situations where $F$ decreases when $x$ gets large imply that pathogens have a direct negative effect on defense production, as Ellner et al. (2021) assumed in their model. Positive values of $\psi$ mean that damage hinders the production of defense. We therefore propose that empirical data indicating bistability, such as those presented in figure 1, suggests that the infection lowers the immune defense either directly, as in Ellner et al. (2021), or indirectly, through damage accumulation or resource diversion, as in van Leeuwen et al. (2019).

Apart from a negative impact of infection on defense production, bistability requires other conditions which involve all parameters. In the following we focused on two parameters which describe the constitutive and the inducible parts of defense production, (namely $\gamma$, the maximum constitutive defense production and $\alpha$, the activation of defense production). In figure $4 \mathrm{~A}$, where the two parameters were varied, two curves delimit a region within which the infection dynamics is bistable. When $\gamma$ is fixed to 2 and only $\alpha$ is varied (which corresponds to the vertical dotted line in figure $4 \mathrm{~A}$ ) the equilibrium load $\tilde{x}$ is very high for low values of $\alpha$ and drops to low values when $\alpha$ is high (fig. 4B). For intermediate values of $\alpha$, the system is bistable: both high load and low load equilibria are possible. The same sort of pattern occurs when $\alpha$ is fixed to 10 and $\gamma$ only is varied (the horizontal dotted line in figure 4A): the equilibrium load is high when $\gamma$ is low, the infection is always cleared when $\gamma$ is high, and the system becomes bistable when $\gamma$ has intermediate values. Inside this bistable region, the high load infection is always possible; the second possible equilibrium is either low load infection, when $\gamma<\gamma_{c}$, or clearance otherwise. 


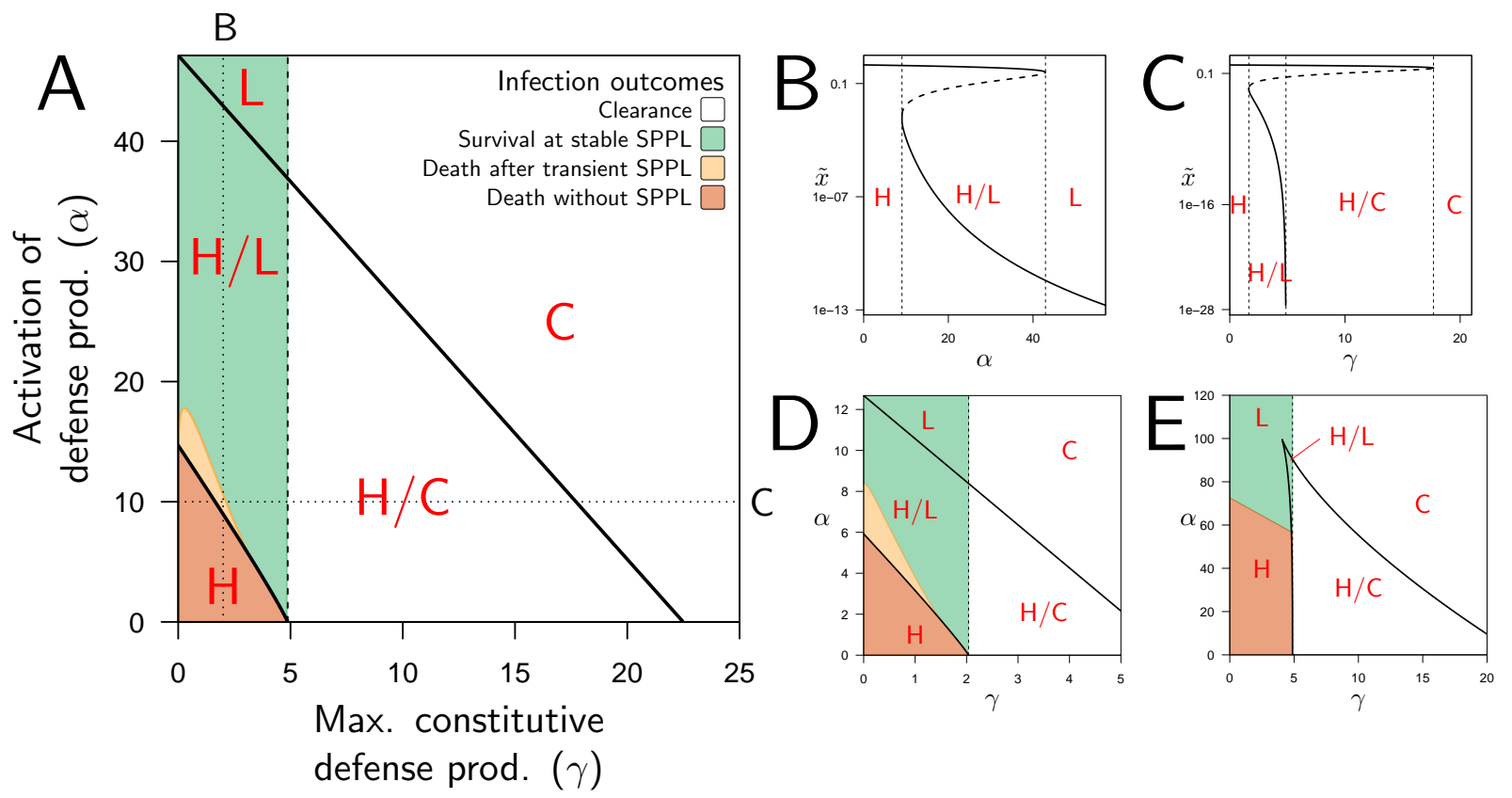

Figure 4: The types of stable equilibria when maximum rate of constitutive defense production $(\gamma)$ and defense activation rate $(\alpha)$ vary. A, parameters are as in figure 2 and both $\alpha$ and $\gamma$ are varied. The vertical dashed line indicates the value of $\gamma_{c}$ above which clearance is stable. Red labels indicate which equilibria are stable: $\mathrm{H}$, high load equilibrium, L, low load equilibrium and $\mathrm{C}$, clearance (the distinction between $\mathrm{H}$ and $\mathrm{L}$ equilibrium is exemplified in figures $\mathrm{B}$ and $\mathrm{C}$ ). The two oblique solid lines delimit a parameter region for which the system is bistable $(\mathrm{H} / \mathrm{L}$ or $\mathrm{H} / \mathrm{C})$. The color indicates which equilibrium the infection actually reaches when the initial load is $x_{0}=10^{-6}$ and when $y_{0}$ and $z_{0}$ are set to the homeostatic state. B. The equilibrium load $\tilde{x}$ as a function of $\alpha$ when parameters are as in A but $\gamma$ is fixed to 2 (which corresponds to the vertical dotted line in A). Solid lines indicate stable equilibria while dashed lines are unstable ones. The distinction between high load and low load equilibria is appropriate here because equilibria with intermediate loads are not stable. C. The equilibrium load $\tilde{x}$ as a function of $\gamma$ with parameters as in A except $\alpha$ fixed to 10 (which corresponds to the horizontal dotted line in A). In the bistable region, the high load equilibrium is always possible; the second possible equilibrium is either low load, when $\gamma$ is below $\gamma_{c}$, or clearance otherwise. D, Parameters as in A except $\psi=0.5$, which reduces the negative impact of damage on defense production. E, Parameters as in A except $u=0.75$, which lowers defense production at low pathogen load.

In figures $4 \mathrm{~B}$ and $\mathrm{C}$, the possible outcomes of the infection can easily be contrasted based on the equilibrium pathogen load: equilibrium load is either high or low, with no possible intermediate situation. This happens because when the system is bistable the two possible stable equilibria are separated by an unstable equilibrium, which the system cannot reach. Red labels indicate which outcome may be observed in figure 4A, with two possibilities when the system is bistable. Coloured areas indicate which outcome is actually observed when the inoculum $x_{0}$ is fixed to $10^{-6}$ and when $y_{0}$ and $z_{0}$ are set to the homeostatic state. When only the high load equilibrium is possible, the host always dies from the infection because the equilibrium level of damage $\tilde{z}$ exceeds $z_{d}$. When only the low load equilibrium is possible, 
the host survives and the infection becomes chronic. In the bistable area, the infection also becomes chronic when clearance is not possible $\left(\gamma<\gamma_{c}\right)$, with load stabilizing at a SPPL. But two situations must be distinguished here: first, when the immunity is strong enough to control pathogens proliferation (the green area in 4A), the infection reaches the low load equilibrium. The host will then survive the infection and load will stabilize at the SPPL for its whole life. Second, when the host immunity is weaker (the yellow area in fig. 4A) defense control proliferation but for a limited period of time only, as in the green curve of figure 3D. The SPPL is therefore transient, and the host will eventually die from the infection, when accumulating damage hinders defense production.

Demonstrating experimentally that a SPPL is transient might prove difficult, first because it is not always feasible to monitor pathogen loads until the host dies and, second, because even if it was, it may still be challenging to demonstrate that the death of an individual host was actually caused by the infection. Our model offers a solution to this problem. Mathematically, a transient SPPL indeed occurs when the three variables progress slowly, because they have approached an equilibrium point where all derivatives $d x / d t, d y / d t$ and $d z / d t$ are close to zero. However, this equilibrium must be unstable, as the trajectories eventually move away from it and head towards a high load infection. This indicates, first, that the SPPL cannot be transient unless an unstable equilibrium point exists with $\tilde{x}>0$, which in turn requires that the system is bistable (this unstable equilibrium corresponds to the dashed curves in fig. $4 \mathrm{~B}$ and $4 \mathrm{C}$ ). Second, it also proves that a transient SPPL is not a true equilibrium situation and, therefore, that the SPPL should vary with initial conditions. We have shown that the SPPL does indeed increase with the initial dose $x_{0}$ when it is transient, while it is independent from initial conditions when stable. Those results are in line with empirical results as the SPPL depends on initial inocculum when Drosophila are infected by pathogenic bacteria (Acuña Hidalgo et al., 2021; Chambers et al., 2019; Duneau et al., 2017a), but not when infected by non-pathogenic bacteria such as E. coli (RamirezCorona et al., 2021). We therefore propose that testing the link between the SPPL and the initial dose is a way to experimentally demonstrate that the control over infection is transient.

Bistability, as we have already discussed, is possible in our model when accumulating damage negatively impacts defense production. As expected, reducing this impact by lowering the value of $\psi$ reduces the range of parameters which permits bistability. With $\psi=2$ in figure $4 \mathrm{~A}, 94 \%$ of the parameter area where the high load equilibrium is possible is bistable; in figure $4 \mathrm{D}$, where $\psi$ is reduced to 0.5 , only $82 \%$ of this same area is bistable. Finally, the distinction between high and low load equilibria, although convenient, is not always possible. In figure $4 \mathrm{E}$, with $u=0.75$, the bistable area forms a closed triangle so that when constitutive defense production $(\gamma)$ are low a continuum exists between high load equilibria, when defense activation $(\alpha)$ is low, and low load equilibria, when $\alpha$ is high. 

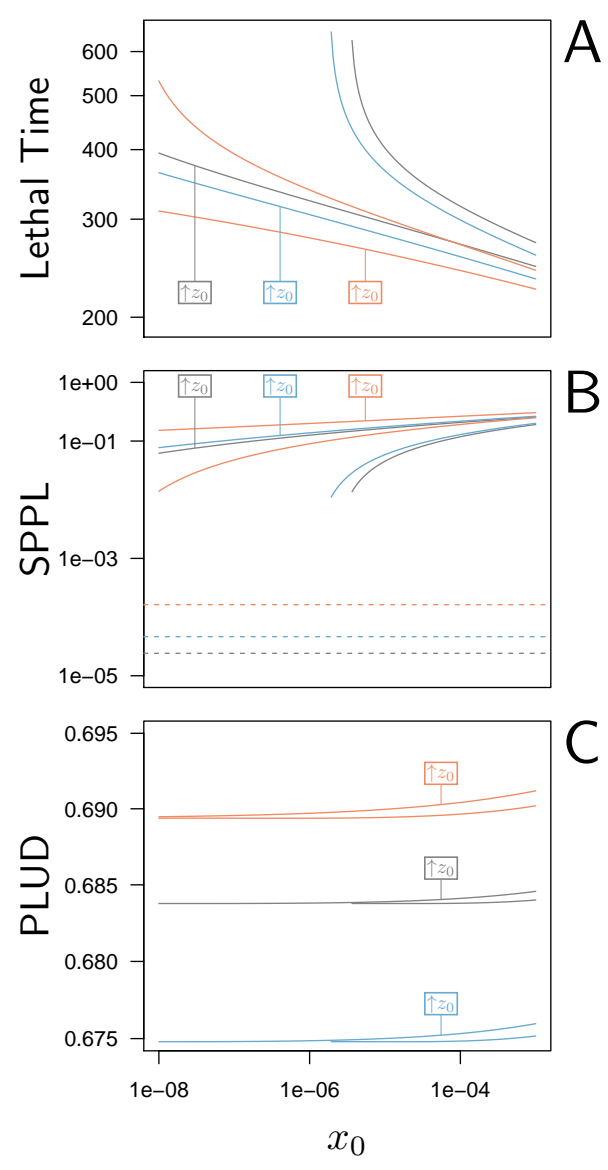

Figure 5: Three quantities that can be experimentally measured on infections, as functions of the inoculated dose. The black curves have been computed with parameters as in fig. 2, while the red curves correspond to a $5 \%$ decrease in defense efficiency $(\delta)$ and the blue ones to a $5 \%$ decrease in damage repair efficiency $(\xi)$. For each parameter set, $y_{0}$ was set at the homeostatic state and $z_{0}$ was either at the homeostatic state or increased by $1 \%$ of $z_{d}$ (indicated by boxes in the figures). A. The Lethal Time (LT) decreases with inoculated dose and with $z_{0}$. Decrease in resistance or in tolerance both reduce the time it takes for the pathogen to kill the host. Note that a decrease in tolerance (blue curves compared to black curves) only shifts the relationship between LT and dose, without altering its slope. B. The stable SPPL (dashed lines) is by definition independent from initial conditions. The transient SPPL (solid lines) conversely increases with both $x_{0}$ and $z_{0}$. Decreased resistance and decreased tolerance both increase the SPPL. Variation in transient SPPL mirror that of LT, which indicates that the SPPL is a good predictor of the host lifespan. C. The PLUD is almost independent from initial dose and slightly increases with $z_{0}$. It increases with lowered resistance (low $\delta$, red curves) but decreases when tolerance is lowered (low $\xi$, blue curves). 


\section{Experimental measures of loads or of mortality al- ways mix tolerance and resistance}

The SPPL and the PLUD can both be estimated from experimental infections, as earlier work by Duneau et al. (2017a) has shown. They both have been proposed to reflect different aspects of the host immune response: the SPPL, stable or transient, being a pathogen load stabilized by the host immunity, could be taken as a proxy for resistance; the PLUD, being the maximum pathogen load the host can endure, could rather reflect the host's tolerance to infection. Measurements of host survival to infection are also commonly used as proxies of resistance or tolerance (e.g. Gupta \& Vale, 2017; Louie et al., 2016). Here, we have used our model to question the way these quantities are used as surrogate measurements for tolerance or resistance. For this purpose, we computed them from simulated infections and surveyed how they relate to immunity parameters (see Supplementary Material S3 and S4 for a complete analysis of the SPPL and the PLUD).

As expected, the time it takes for a pathogen to kill its host decreases if inoculum size is increased (fig. 5A) or if the host is wounded prior to infection (i.e. when $z_{0}$ is increased by $1 \%$ of $z_{d}$ above the homeostatic state). Lethal Time (LT) thus strongly depends on the conditions in which the infection has been initiated. Our simulations further show that any genetic change in the host or the pathogen that reduces the host resistance (red curves in fig. 5A) or tolerance (blue curves in fig. 5A) accelerates death. We also showed that contrary to what some authors have proposed (e.g. Gupta \& Vale, 2017; Louie et al., 2016), a reduction in tolerance does not modify how LT relates to dose; a reduction in resistance, conversely, makes LT less dependent on dose (compare red and blue curves in fig. 5A). Hence, the comparison of lethal time in response to different doses is more likely to characterise differencies in resistance than in disease tolerance.

The SPPL, when it is stable, does not depend on initial conditions (dashed lines in figure 5B). This is because a stable SPPL is an equilibrium towards which the infection will converge, no matter where it starts from, as long as the host survives the infection. The transient SPPL, conversely, is not an equilibrium. We showed that it does increase with dose and is higher when the host is wounded prior to infection. Our simulations finally indicate that both transient and stable SPPL increase when either tolerance or resistance are impeded (see Supplementary Material S1 and S3 for a complete analysis). In fact, if by definition stable SPPL does not correlate with the time the host will die, variations in the transient SPPL almost exactly mirror that of the LT. This is because the control of proliferation does not last long when the transient SPPL is high: infections which maintain high pathogen loads rapidly progress towards host death. A first practical consequence of this observation is that measurements of the SPPL and of the LT bear the same information on the host immunity, so that it is useless to measure both, unless one wants to predict transmission rate, which should quantitatively depend on both bacterial load and infection duration. A second practical consequence is that the SPPL, when transient, can be used as a predictor of the host's lifespan, as already demonstrated in HIV infections (Fraser et al., 2007; Mellors et al., 1996).

The two hosts of figures 3D-F die at very different times but at almost indistinguishable PLUD. This is confirmed by figure 5A, where the PLUD does not vary with changes in 
inoculum size that do otherwise yield three-fold variations in LT (compare fig. 5C to fig. 5A). More generally, the PLUD is only weakly influenced by the conditions in which the infection has been initiated, albeit we predict that it should slightly increase when the host is wounded. Our model therefore reproduces an important property of the PLUD which Duneau et al. (2017a) have documented (see fig. 1): for a given pair of host and bacterial pathogen, the PLUD in D. melanogaster is almost constant and does not correlate with the time to succumb from the infection. In our model, this comes from the fact that the pathogen load $x$ evolves much faster than other variables at the start of the infection (as detailed in Supplementary Material S4). Pathogens thus rapidly reach the highest possible load allowed by the current amount of defense $\left(x_{t} \lesssim 1-\delta y_{t}\right)$. The dynamics of $x$ is then "enslaved" by that of $y$ and $z$ (van Kampen, 1985) and, as the disease enters its final stage, load increases slowly while $y$ gradually diminishes under the effect of accumulating damage. Because of this very particular dynamics, the way $x_{t}$ relates to $z_{t}$ will be very similar for all infections that enter their final phase, which in the end renders the PLUD almost independent from the conditions that prevailed at the onset of the infection.

Another particularity of the PLUD, is that it increases when resistance is diminished (red curves in fig. $5 \mathrm{C}$ ) but increases with tolerance (blue curves in fig. $5 \mathrm{C}$ ). The PLUD is therefore a composite measurement which reacts to both resistance and tolerance traits, just like the SPPL and the LT, but it has the unique feature that it can respond differently to these two types of variation (see Supplementary Material S4 and the following section for a more complete elasticity analysis).

\section{Combining PLUD and Hazard Ratio (HR) to char- acterize the host's ability to handle infections}

We have seen before that variations in resistance and variations in disease tolerance have distinctive impacts on the PLUD. Still, a host lineage with higher than average PLUD could either be less resistant or more tolerant than other lineages. The PLUD is therefore difficult to interpret by itself, but we shall demonstrate that its relationship with mortality measurements is informative. To investigate this, we performed elasticity analyses of the PLUD and of the Hazard Ratio, a quantity which measures death risk relative to a chosen reference host (HR, see Supplementary Material S5 for definitions and analyses). As our model allows to determine the time to death of a given infection, the HR can be obtained from a Cox proportional hazard model (Cox, 1972) adjusted on simulated survival curves.

As expected, we found that reducing the risk to die from the infection can be achieved through a tighter control of pathogen proliferation or, alternatively, by mitigating the damage the infection causes (fig. 6A and 6B). For example, HR decreases both when the defense is made more efficient ( $\delta$ increases) and when damage repairing is accelerated ( $\xi$ increases).

Variations in PLUD are more complex, but figure 6A suggests that reducing damage (e.g by increasing damage repair, $\xi$ ) or making the host more damage-tolerant (by increasing the maximum damage level the host can sustain, $z_{d}$ ) should increase the PLUD. As a result, variations in parameters which have a direct impact on the dynamics of damage produce a negative correlation between PLUD and HR. Figure $6 \mathrm{~A}$ shows that the correlation that 

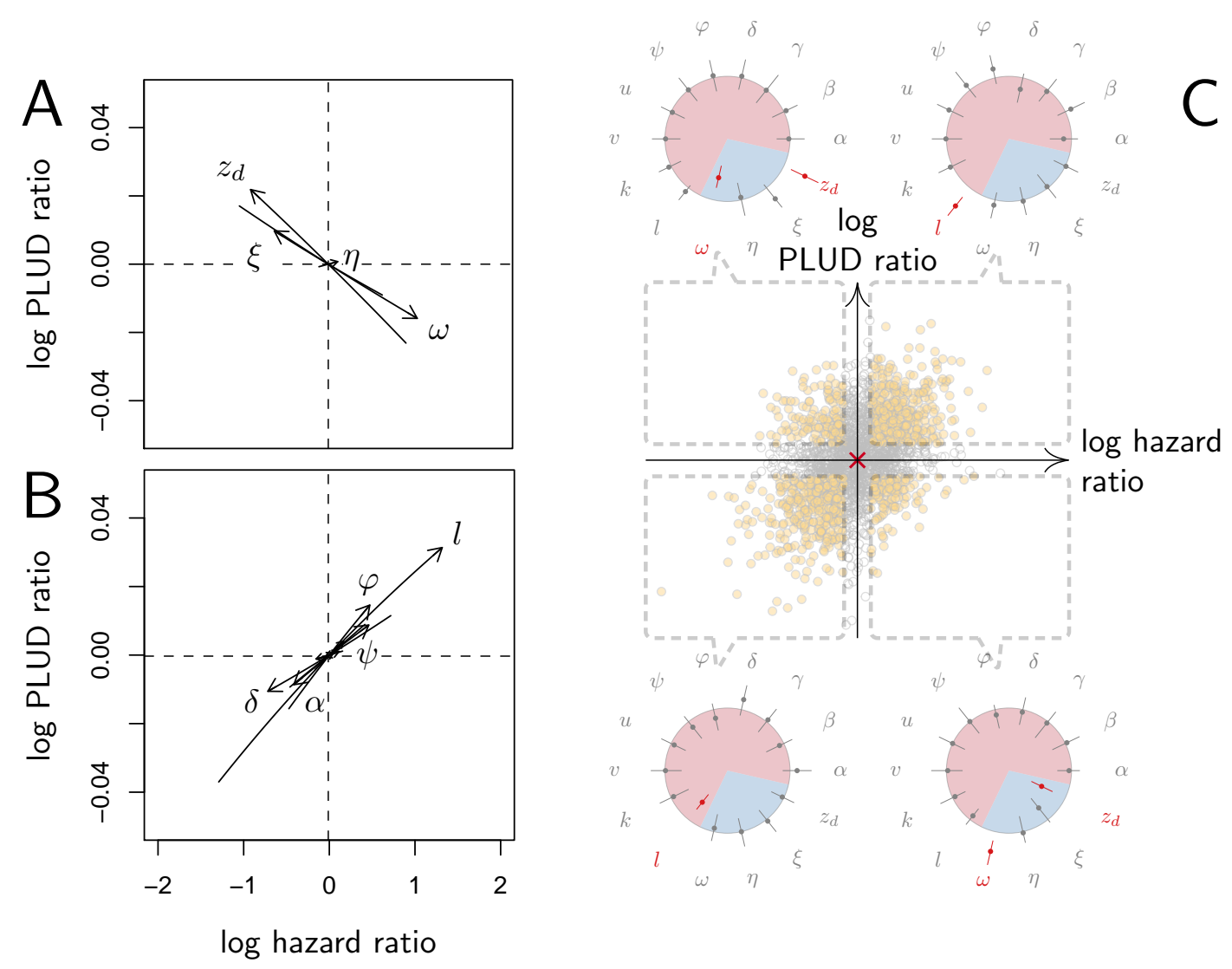

Figure 6: Effect of parameter variations on PLUD and HR. In A-B, parameters are initially fixed as in figure 2 except for $\gamma=0.5$, as in red curve if fig. 3. Parameters are then varied one at a time, from $-5 \%$ to $+5 \%$. For each modified parameter value, the PLUD was computed and 100 survival simulations were run with $x_{0}$ randomly drawn from a log-normal distribution with average $10^{-6}$ and variance 0.5. A Cox proportional hazard model was then fitted on simulated data so that log Hazard Ratio (log HR) could be related to parameter variation. The horizontal dashed lines would correspond to parameters which have no influence on PLUD; the vertical dashed line would correspond to those which do not impact HR. A The effect of tolerance parameters (defined as in figure 2A). Arrows indicate that, as expected, any increase in the damage induced by the pathogen $(\omega)$ decrease the PLUD, while increasing damage repair $(\xi)$ or tolerance to damage $\left(z_{d}\right)$ has the opposite effects. For the parameter set we have used here, increasing $\eta$ has almost no effect on PLUD and only slightly increases HR. B The effects of resistance parameter (defined as in figure 2A). Variation in any of these parameters produces a positive correlation between PLUD and HR. C The variation in PLUD and HR when all parameters are all randomly drawn from independent Gaussian laws. The red cross corresponds to a strain with parameters as in fig. 2 and each dot is a "mutant strain" which parameters have $95 \%$ chances to deviate by less than $0.5 \%$ from that of the "wild-type strain". Open circles are mutants which either log PLUD ratio or log HR ratio do not significantly deviate from zero (see text for details). We sorted mutants in four groups, according to whether their PLUD and HR has significantly increased or decreased compared to the wild strain. The distributions of traits are indicated for each group on colored circles, with segments spanning $75 \%$ of the deviation range and the central dot corresponding to the median deviation. Segments in red indicate that $75 \%$ of the mutants have either lower trait values than the wild-type strain (when the segment is inside the circle) or higher trait values (when outside). 
results from variations in pathogenicity $(\omega)$ is almost indistinguishable from that produced by variations in damage repair $(\xi)$, and close to that produced by variations in the maximum level of damage the host can sustain $\left(z_{d}\right)$. This means that experimental measurements of PLUD and HR would probably not allow to distinguish the consequences of variations in the host's ability to repair and the variation in level of damage it can sustain. Only variations in $\eta$, which measures the damage caused by defense, have a distinct signature as they have almost no effect on PLUD.

Figure 6B further suggests that anything that reduces pathogen proliferation, like increasing the production or efficacy of defense $(\alpha$ or $\delta$ ), comes with a decreased PLUD. Variations in parameters that control the defense therefore result in a positive correlation between the PLUD and HR.

In summary, figure 6 indicates that measuring the co-variation of the PLUD and the HR could help to experimentally determine if better survival is achieved by greater tolerance or rather by higher resistance.

The case of $\eta$, which quantifies the importance of defense side effects, demonstrates that although appealing, this method has its limits. This parameter clearly relates to damage production and we demonstrate that the lower bound of the PLUD expectedly decreases when $\eta$ increases (see equation (S4-1) in Supplementary Material S4). But when immunity is weak, this effect can reverse with increasing $\eta$ impairing control and resulting in higher PLUD (as we demonstrate in our elasticity analysis, see figure S4-2 when both $\alpha$ and $\gamma$ are low). In hosts with very weak immunity, therefore, variations in the detrimental side-effects of defense $(\eta)$ can create a positive correlation between PLUD and HR (as in figure 6A). This raises a necessary caution while studying the PLUD in immunodeficient individuals.

The practical use of the above proposed method will largely depend on which specific parameters differ the most among the hosts and pathogens to compare. We cannot predict much on this matter, as everything will obviously depend on the genetic variation of these traits. A crude way to test the utility of the method, though, is to let all parameters vary and see which effect predominates. In figure 6C, we randomly drew all 14 parameters from independent Gaussian laws with average set at the parameter values given in figure 2, and a variance chosen so that $95 \%$ of the random values differ by less than $0.5 \%$ from this average. If we consider that the parameter set given in figure 2 represents a wild-type host, each random parameter set can be considered as being a mutant strain. We randomly sampled 2,000 such mutants and simulated 100 survival curves for each of them. We compared their HR to that of the wild-type using a Cox proportional hazard model. We compared their PLUD to that of the wild-type as if load was estimated by plating. For that purpose, we randomly drew numbers of Colony Forming Units (CFU) from Poisson distributions with average given by the predicted WHD, which we then compared between the mutant and the wild-type hosts using a Poisson glm. We have drawn one number of CFU per host from a Poisson distribution with average 1000 times the simulated PLUD, so that we have similar statistical powers when comparing PLUDs and HRs. In figure $6 \mathrm{C}$, the red cross lying on the origin is the wild-type; mutants which significantly deviate from it (closed circle) are separated in four groups, according to the deviation signs. For each group, we compared the distribution of mutant traits to that of the wild-type. We found that mutants with significantly higher HR and PLUD have almost systematically a stronger down-regulation of defense production (with clearly higher value of $l$ ) and a slightly lower efficiency of defense 

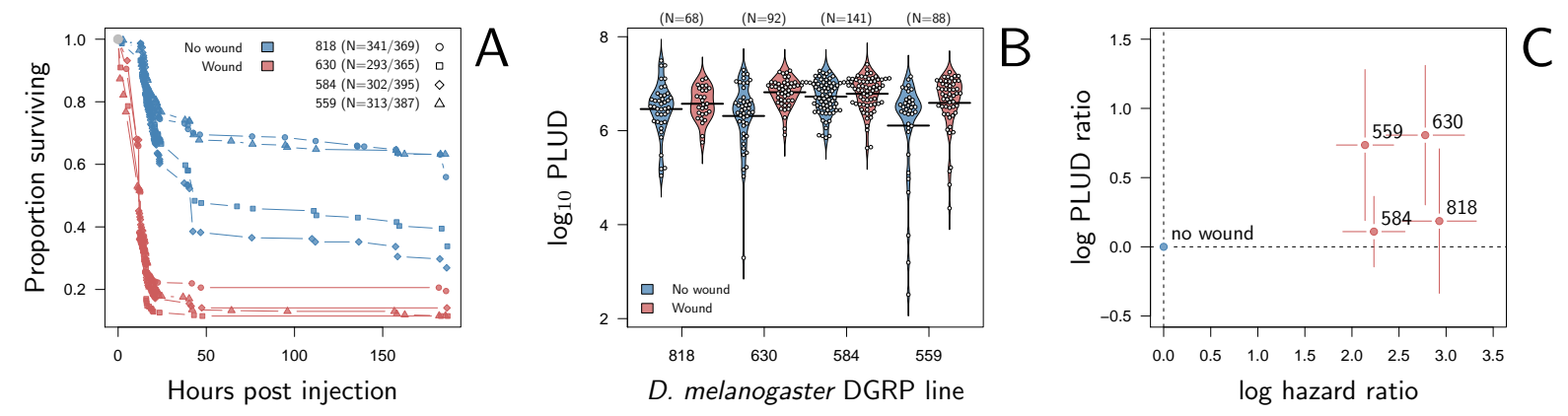

Figure 7: HR and PLUD estimations on D. melanogaster infected with Providencia rettgeri with or without thorax wound prior to injection. We performed the experiment on four genotypes sampled from the Drosophila Genetic Reference Panel (lines RAL-818, RAL-630, RAL-584 and RAL-559). A. Proportion of surviving flies as a function of hours post injection. Numbers in legend indicate sample sizes for no wound/wound treatments, respectively. In all lines, the wound significantly and sharply reduces survival. B. PLUD for each D. melanogaster line. Each point represents an individual fly and the bars represent the means. C. Log PLUD ratio as a function of log Hazard Ratio. For each line, we computed the log-ratio of PLUD in wounded flies to that in non-wounded, the value of zero therefore corresponding to a non-wounded reference as for log-HR. Bars represents the $95 \%$ CI as calculated by 1.96 times the standard error. The wound constantly increases both the HR and the PLUD, as our model predicts, although clearly significant variations are found in lines RAL-630 and RAL-559 only.

$(\delta)$. Hence, such mutants die faster than the wild-type because they are less resistant. Mutants with higher HR but lower PLUD have most often a lower tolerance to damage (lower $z_{d}$ ) and are more susceptible to the pathogen virulence (greater $\omega$ ). These mutants therefore die faster than the wild-type strain because they are less disease tolerant.

\section{Wounding Drosophila melanogaster or suppressing some of its active effectors both increase the PLUD}

Our model predicts that when damage hinder defense production, hosts have higher PLUD if wounded prior to infection (see fig. $5 \mathrm{C}$ ). We tested this prediction by measuring the PLUD of the bacterial pathogen Providencia rettgeri injected in D. melanogaster that were either injured in the thorax before being injected in the abdomen or not injured (see fig. 7 and Supplementary Material S6 for methodological details). As expected, the wound does always significantly reduce survival (Cox proportional hazard model with random experimental blocks: $\chi^{2}=1397.44, d f=1, \mathrm{p}-$ value $\left.=7.5 \times 10^{-306}\right)$. In two of the four replicates $D$. melanogaster isogenic lines, the PLUD is significantly higher in wounded animals than in non-wounded while in the two other lines, the wound-induced increase in PLUD is nonsignificant. When analyzing all lines together, we found a significant effect of wound on PLUD (negative binomial GLMM with random experimental blocks: $\chi^{2}=14.73$, $d f=1$ $\mathrm{p}-$ value $=0.00012)$ which indicates that overall the wound does increase both hazard ratio 

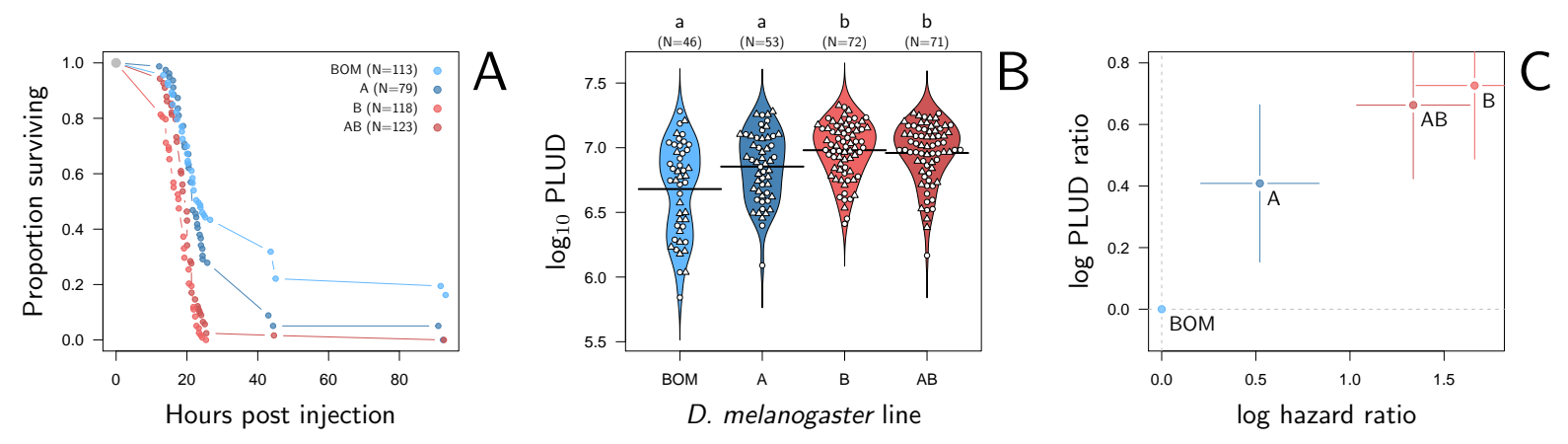

Figure 8: HR and PLUD estimations on D. melanogaster immuno-deficient lines infected with Providencia rettgeri. A. Proportion of surviving flies as a function of hours post injection. All lines significantly differ(Cox proportional hazard model, $p<0.012$ ), but lines B and AB, which both lack Diptericins, are much more susceptible than A, which lacks Defensin only, and BOM, which lacks Bomanins only, both types of effectors less efficient against $P$. rettgeri infections. B. The estimated PLUD by individual from each line (BOM, A, B and AB). Triangles and circles correspond to two replicate experiments. Small letters above indicate significant differences, as tested by a pairwise Wilcoxon test. Lines B and AB have a significantly higher PLUD than A and BOM. Black lines represent the means. C. Log PLUD ratio as a function of log HR. All ratios are here computed relative to the BOM line (indicated by the blue dot on the origin). Bars represents the $95 \%$ CI as calculated by 1.96 times the standard error. Loss-of-function of active immune effectors increases both the HR and the PLUD, as our model predicts.

and PLUD, as our model predicts. This result can be taken as an evidence that inflicting damage to D. melanogaster hinders the production of defense, and thus indirectly reduces resistance.

Our model also predicts that mutations that decrease resistance should in most cases increase the PLUD. We tested this prediction by infecting D. melanogaster mutants from which some effectors (e.g. antimicrobial peptides) are lacking (see Supplementary Material S6 and (Hanson et al., 2019) for a description of mutants). This way we impeded the immune system by affecting immune effectors, the most direct actors of resistance mechanisms. We used isogenic lines sharing the same genetic background but from which genetic deletions have induced a loss-of-function in: Bomanins (line BOM); Defensin (line A); or Drosocin, Attacins and both Diptericins (line B). A fourth mutant line (line AB) combines the loss-of-function of lines A and B. Bomanins are Toll induced defense which are largely activated by infection with Gram-positive bacteria and fungi (Clemmons et al., 2015). They have little effect on Gram-negative such as P. rettgeri (Hanson et al., 2019). Thus, we have used the BOM line as a control carrying mutations but from which we know the phenotype on resistance would be limited. Line A lacks Defensin, an antimicrobial peptide strongly upregulated by P. rettgeri infection (Duneau et al., 2017b; Troha et al., 2018) but from which the mutation has a limited effect on survival (Hanson et al., 2019). Lines B and AB, which both lack Diptericins, are conversely expected to be highly susceptible to P. rettgeri (Hanson et al., 2019; Unckless et al., 2016). We found that, compared to lines BOM and A, lines B and AB have higher death rates when infected by P. rettgeri (fig. 8A; Cox proportional hazard 
model with random experimental blocks: $\chi^{2}=138.12, d f=3, \mathrm{p}-$ value $\left.=9.6 \times 10^{-30}\right)$. We also found that these same two lines had highest PLUD (fig. 8B; negative binomial GLMM with random experimental blocks: $\chi^{2}=43.71, d f=3, \mathrm{p}-$ value $\left.=1.73 \times 10^{-9}\right)$. The higher PLUD of $\mathrm{B}$ and $\mathrm{AB}$ lines therefore reveals their low resistance to $P$. rettgeri infections and PLUD ratio positively correlates with HR, as our model predicts (fig. 8C).

\section{Discussion}

Signs and symptoms of infectious diseases are highly variable: severe diseases yield a certain and rapid death when untreated, while benign diseases remain almost unnoticed. The load pathogens can reach is key in determining both the effect and the outcome of an infection and it therefore explains part of this variation. What will in turn determine load depends not only on environmental conditions, but also on various and interacting genetically fixed characteristics of both the host and the pathogen. This complex and intricate system is commonly simplified by considering that the host and pathogen traits fall in two categories: those which determine the resistance to the infection, i.e. how efficiently pathogen proliferation is controlled by immunity, and those which allow to tolerate the damage inflicted by the infection. We have built a process based model of Within Host Dynamics (WHD) of pathogen to explore these notions of resistance and tolerance. The method we have used is to study how the proxies employed in literature to quantify them connect to the processes our model encompasses.

To ensure this approach is possible, our model behaviour needs to match experimental evidence. We first found that it recreates the most commonly observed types of WHD documented to date in D. melanogaster and in other insect hosts system. The model thus provides a general theoretical framework which can be useful to investigate most situations of experimental infection. Most importantly, it finely reproduces the properties of two experimental proxies which have been used to evaluate resistance or tolerance to disease: the Set-Point Pathogen Load (SPPL) increases with dose in chronic lethal infections but not in chronic benign infections; the Pathogen Load Upon Death (PLUD) increases if the host is wounded before infection but is insensitive to inoculum size. We take these results as evidence that the biological basis on which we grounded the model are sound.

We shall in the following section summarize how the SPPL and the PLUD relate to model parameters and how they can yield insights into resistance and tolerance. A general conclusion is that they reflect both mechanisms. We were nevertheless able to predict what information can be gained from each of them and propose experimental methods to tease apart resistance and tolerance effects.

\section{Exploring chronicity: stable and unstable Set-Point Pathogen Loads}

When a host survives the infection but does not successfully clear the pathogen, the disease enters a phase of chronicity (Virgin et al., 2009) and load stabilizes at a Set-Point Pathogen Load (SPPL). Such chronic phases characterize a large range of infectious diseases, and we have shown that this is determined by the host immune defense. Notably, strong constitutive defense prevents chronicity because it permits the host to clear pathogens; weak constitutive defense combined to slow activation upon pathogen detection also prevents chronicity because 
hosts then die from the infection.

A more surprising prediction of our model is that chronicity can be transient, the SPPL being then unstable. This happens when the damage which accumulate during the infection eventually obstruct the production of immune defense. Pathogen proliferation is then unleashed which leads to host death. Illustrations of such infections could be, in humans, $M y$ cobacterium tuberculosis causing tuberculosis infections or HIV causing AIDS; in Drosophila, this could be Providencia rettgeri infections. When the SPPL is stable, by contrast, the host never clears pathogens but it will not die either from the infection. In human, this could be Porphyromonas gingivilis causing gum infections; in Drosophila, this could be Escherichia coli infections. Our model clearly highlighted those two types of chronic infections and suggests that their SPPL cannot be interpreted the same way.

In particular, we found that unstable SPPLs depend on how the infection was initiated. For example, we predict that they should be higher when the host is injured prior to contamination and should correlate positively with the inoculum size (see fig. 5). These predictions agree with previous study of bacterial infection of D. melanogaster (Acuña Hidalgo et al., 2021; Chambers et al., 2014, 2019; Duneau et al., 2017a). Our model predicts that stable SPPLs are, conversely, independent from how the infection is initiated. In particular, they are insensitive to inoculum size, in accordance with benign Escherichia coli infections of $D$. melanogaster (Ramirez-Corona et al., 2021). The stable SPPL therefore reflects stable genetically determined characteristics of both the host and the pathogen, but it is affected by traits related to both tolerance and resistance. For example, low stable SPPL could indicate either that the host has efficient immune defense, or that it has impeded tolerance.

This is not to say that no information can be gained from the study of SPPL, be it stable or unstable. In probably all chronic infections, everything being equal, the pathogen load during the chronic phase is an appropriate proxy to compare the capacity to be transmitted to another host: the higher the SPPL, the most the host sheds pathogens (Fraser et al., 2007; Matthews et al., 2006). The unstable SPPL is also an important concept because it is a good predictor of the duration of chronicity: we found indeed that the higher the SPPL, the earlier the host dies from the infection. Monitoring survival to infection and transient SPPL is therefore providing redundant information. This is particularly well established in the specific case of HIV infections, where high SPPL is associated to short asymptomatic phases and rapid progression to the final stage of the disease (Fraser et al., 2007). The SPPL in HIV infections has also raised considerable attention because of two striking characteristics which are not fully understood. First, it is highly variable among patients; second, it is heritable, which means that the SPPL of a newly infected patient positively correlates to that of the person it has been infected by. The most common explanation of this correlation is that part of the variation in SPPL is caused by genetic variation in viruses. But if variance in SPPL has a genetic origin, the SPPL should evolve during the course of the infection and load should therefore increase over time. The heritable nature of SPPL therefore contradicts the observation that load is constant throughout the asymptomatic phase of the disease (although some possible solutions to this paradox have been proposed Bonhoeffer et al., 2015; Hool et al., 2014). Our model suggests that part of the large variance in SPPL could be non-genetic yet heritable. If transmission events occur during chronicity, the inoculum size should indeed increase with the SPPL of the donor host (as suggested by Fraser et al., 2007). As we have demonstrated that the transient SPPL does increase with inoculum size, 
the SPPL of a newly contaminated host should reflect that of the donor host. The fact that the transient SPPL increases with inoculum size could therefore suffice to create a form of non-genetic heritability in SPPL.

Finally, we propose that quantifying the SPPL for various doses in a chronic disease would allow, if the SPPL increases with dose, to demonstrate that it is unstable. This could be used as an experimental method to prove that chonicity is transient. It would in addition demonstrate that the system is bistable (a necessary condition for the existence of unstable SPPL) which in turn requires that the infection impairs the production of defense either directly (as in Ellner et al., 2021) or indirectly, through the accumulation of damage (Stoecklein et al., 2012; van Leeuwen et al., 2019). Hence, showing that the SPPL is transient informs on the fact that the pathogens, or the damage they cause, obstruct the immune response.

\section{Characterizing lethal diseases: the Pathogen Load Upon Death}

The Pathogen Load Upon Death is the maximum pathogen load a host can sustain before it dies from the infection. It has been logically used as a measure of disease tolerance (Duneau et al., 2017a; Huang et al., 2020; Troha et al., 2018) and of pathogenicity (Faucher et al., 2021). As expected, our model confirms that the PLUD strongly depends on tolerance to damage $\left(z_{d}\right.$, see Supplementary Material S4). However, if the PLUD was an unambiguous quantification of tolerance, it should not depend on any other parameter than $z_{d}$, or at least not on parameters that determine the control of pathogen proliferation. Unlike what was previously suggested (Duneau et al., 2017a), but in agreement with our results obtained with a more powerful experimental design (fig. 8B), hosts with an impeded immune response are expected to have a higher PLUD. For instance, in our model, the PLUD increases when less defense are produced (e.g. when $\alpha$ or $\gamma$ decrease, see fig. S4-2) or when defense are made less efficient (i.e. when $\delta$ is decreased, see figure 6). This is because the PLUD is a load which, as such, reflects the ability of pathogens to proliferate inside the host. Hence, like the transient SPPL and probably like any quantity computed from load, the PLUD is subject to mixed influences of immune control of proliferation and of damage mitigation.

The PLUD, unlike the transient SPPL, is independent from inoculum size: flies injected with large inoculum die faster but at the same load than flies infected with small inoculum (Duneau et al., 2017a). Our model reproduces this observation, and we have demonstrated mathematically that this happens because pathogen load is a "fast variable" (van Kampen, 1985, see Supplementary Material S4). In practice, once the accumulation of damage due to the infection is starting to affect the immune system in a way that the defense are impeded, the system is entering a run-away process: pathogens proliferate faster, inflicting new damage to the host, which decrease the defense further. Infections that enter this dynamics tend to all follow the same typical trajectory, and therefore kill the host at the same pathogen load. We found that this occurs in most parameter combinations (see Supplementary Material S4), except when hosts die at very low damage (i.e. when $z_{d}$ is low). In that particular case, death occurs so rapidly that infections have no time to converge to their typical final trajectory and the PLUD depends on inoculum size. This could represents situations where the host is already extremely weak or about to die. However, in most relevant cases, the PLUD being independent on how the infection has started, it reflects the genetically fixed traits of both hosts and pathogens that influence disease tolerance and resistance. 
The PLUD as an aid to compare tolerance and resistance among hosts or diseases

It has been proposed that disease tolerance could be quantified as the slope of a linear relation of time to death to a load measured at a fixed point during the infection (e.g. Louie et al., 2016; McCarville \& Ayres, 2018) or with the linear or logistic relationship between initial inoculum size and time to death (e.g. Gupta \& Vale, 2017). However, our analysis suggests strongly that these approaches mostly measures resistance (see fig. 5). This is probably due to the fact that any pathogen load is subjected to resistance mechanisms. Instead, we propose to use the Hazard Ratio and the PLUD to determine whether difference in susceptibilities are mainly due to default in tolerance or resistance mechanisms. The method is somewhat similar but differs in that, firstly, we propose to use a load that reflect a moment of the infection instead of a load measured at a fixed, arbitrary, time of the infection. This ensures that these loads can be compared among hosts and among pathogens as they always have the same biological interpretation. Secondly, we used measures of Hazard Ratio obtained for a fixed inoculum size, because we found that changing inoculum size has little impact on the relation between the PLUD and the Hazard Ratio, and because high inoculum can lead to artifacts as it might artificially by-pass immune responses.

Observing that a particular lineage of host has a lower than average PLUD cannot indicate whether this lineage is less tolerant or more resistant than others to the infection. However, our model shows that if this low PLUD lineage also has a higher than average risk to die from the infection (or a shorter time to death) we can conclude that its susceptibility is largely due to a default in tolerance to infection. If conversely a lineage has a higher than average risk to die with a higher PLUD, we would conclude that its susceptibility is due largely to a default in resistance (see fig. 6).

We confirmed this prediction in the case of P. rettgeri infecting D. melanogaster: host genotypes with impeded immune effectors have both a higher Hazard Ratio and a higher PLUD than host genotypes with a more efficient immune response. We could not replicate the approach for tolerance because so far no tolerance gene is unambiguously identified in D. melanogaster, probably in part because damage are uneasy to quantify experimentally. CrebA and Bombardier are candidate genes for tolerance in D. melanogaster (Lin et al., 2020; Troha et al., 2018), but they have been determined as such using the PLUD as a tolerance proxy, which would make our reasoning circular. Other genes such as grainyhead, debris buster and $G 9 a$, are suggested to have a role in disease tolerance, but the studies used methods of which we discussed the limits (Howick \& Lazzaro, 2017; Merkling et al., 2015). In fact, the role of G9a in controlling bacterial load is still debated (Gupta \& Vale, 2017). This part of validation therefore remains to be done, but we take the fact that our model reproduces all the documented properties of both the SPPL and the PLUD as a sign that it reflects the biology of the system. We thus propose that a negative correlation between Hazard Ratio and PLUD can be used to detect a default in tolerance, which opens the possibility to determine the genes involve in this part of immune response.

One of the great benefit of theoretical studies is that they allow to explore notions which arose from empirical work. Concepts which seem well and clearly defined may often be underlain by complex and intermingled mechanisms. Our approach has helped to clarify the notions of disease tolerance and disease resistance, in particular by showing how experimental measurements can be misleading when interpreted as unambiguous measurements of one or the other mechanism. Many other mechanisms which determine important aspects of 
infection pose the same sort of difficulty. For example, the co-regulation of damage repair and immune response is now well accepted (Martins et al., 2019; Pucillo \& Vitale, 2020) but remains difficult to study. We advocate that pursuing this combination of theoretical and empirical approaches is needed first to predict how such intermingled mechanisms impact the infection outcomes, and second to design appropriate proxies and experimental methods to quantify their impact.

Acknowledgments We thank Jennifer Regan and Helen Alexander for their comments on the manuscript. The project was supported by the French Laboratory of Excellence project "TULIP" (ANR-10-LABX-41 and ANR-11-IDEX-0002-02), and by the LIA BEEG-B (Laboratoire International Associé-Bioinformatics, Ecology, Evolution, Genomics and Behavior) (CNRS). PF was partly supported by the Darwin Trust of Edinburgh PhD studentship.

Competing interest Authors declare no competing interest.

\section{References}

Acuña Hidalgo, B., Silva, L. M., Regoes, R. R., Franz, M., \& Armitage, S. A. O. (2021). Decomposing virulence to understand bacterial clearance in persistent infections. bioRxiv, 2021.03.29.437521. https://doi.org/10.1101/2021.03.29.437521

Ayres, J. S., \& Schneider, D. S. (2008). Two ways to survive an infection: What resistance and tolerance can teach us about treatments for infectious diseases. Nature Reviews Immunology, 8, 889-895. https://doi.org/10.1038/nri2432

Barda, N., Riesel, D., Akriv, A., Levy, J., Finkel, U., Yona, G., Greenfeld, D., Sheiba, S., Somer, J., Bachmat, E., Rothblum, G. N., Shalit, U., Netzer, D., Balicer, R., \& Dagan, N. (2020). Developing a COVID-19 mortality risk prediction model when individual-level data are not available. Nature Communications, 11, 4439. https://doi.org/10.1038/s41467-020-18297-9

Bonhoeffer, S., Fraser, C., \& Leventhal, G. E. (2015). High heritability is compatible with the broad distribution of set point viral load in hiv carriers. PLoS Pathogens, 11(2). https: //doi.org/10.1371/journal.ppat.1004634

Buchon, N., Silverman, N., \& Cherry, S. (2014). Immunity in Drosophila melanogaster - from microbial recognition to whole-organism physiology. Nat Rev Immunol, 14, 796-810. https: //doi.org/10.1038/nri3763

Chambers, M. C., Jacobson, E., Khalil, S., \& Lazzaro, B. P. (2014). Thorax injury lowers resistance to infection in Drosophila melanogaster. Infection and Immunity, 82(10), 4380-4389. https: //doi.org/10.1128/IAI.02415-14

Chambers, M. C., Jacobson, E., Khalil, S., \& Lazzaro, B. P. (2019). Consequences of chronic bacterial infection in Drosophila melanogaster. PLoS One, 14(10), 1-22. https://doi.org/ 10.1371/journal.pone.0224440

Chang, W., Cheng, J., Allaire, J., Sievert, C., Schloerke, B., Xie, Y., Allen, J., McPherson, J., Dipert, A., \& Borges, B. (2021). Shiny: Web application framework for $r$ [R package version 1.6.0]. https://CRAN.R-project.org/package=shiny

Clemmons, A. W., Lindsay, S. A., \& Wasserman, S. A. (2015). An effector peptide family required for Drosophila Toll-mediated immunity. PLoS Pathogens, 11(4), 1-17. https://doi.org/10. 1371/journal.ppat.1004876

Colaço, H. G., Barros, A., Neves-Costa, A., Seixas, E., Pedroso, D., Velho, T., Willmann, K. L., Faisca, P., Grabmann, G., Yi, H.-S., Shong, M., Benes, V., Weis, S., Köcher, T., \& Moita, 
L. F. (2020). Tetracycline antibiotics induce host-dependent disease tolerance to infection. Immunity. https://doi.org/https://doi.org/10.1016/j.immuni.2020.09.011

Cox, D. R. (1972). Regression models and life-tables. Journal of the Royal Statistical Society, Series B., 34 (2), 187-220. https://www.jstor.org/stable/2985181

Duneau, D., Ferdy, J.-B., Revah, J., Kondolf, H., Ortiz, G. A., Lazzaro, B. P., \& Buchon, N. (2017a). Stochastic variation in the initial phase of bacterial infection predicts the probability of survival in D. melanogaster. eLife, 6, e28298. https://doi.org/10.7554/eLife.28298

Duneau, D., Kondolf, H. C., Im, J. H., Ortiz, G. A., Chow, C., Fox, M. A., Eugenio, A. T., Revah, J., Buchon, N., \& Lazzaro, B. P. (2017b). The Toll pathway underlies sexual dimorphism in resistance to both Gram-negative and positive-bacteria in drosophila. BMC Biology, 15(1), 124. https://doi.org/10.1186/s12915-017-0466-3

Ellner, S. P., Buchon, N., Dörr, T., \& Lazzaro, B. P. (2021). Host-pathogen immune feedbacks can explain widely divergent outcomes from similar infections. Proceedings of the Royal Society B, 288(1951), 20210786. https://doi.org/10.1098/rspb.2021.0786

Faucher, C., Mazana, V., Kardacz, M., Parthuisot, N., Ferdy, J.-B., \& Duneau, D. (2021). Stepspecific adaptation and trade-off over the course of an infection by gasp mutation small colony variants (P. Keim, Ed.). mBio, 12(1). https://doi.org/10.1128/mBio.01399-20

Ferrandon, D. (2013). The complementary facets of epithelial host defenses in the genetic model organism Drosophila melanogaster: From resistance to resilience. Current Opinion in Immunology, 25(1), 59-70. https://doi.org/10.1016/j.coi.2012.11.008

Fraser, C., Hollingsworth, T. D., Chapman, R., de Wolf, F., \& Hanage, W. P. (2007). Variation in HIV-1 set-point viral load: Epidemiological analysis and an evolutionary hypothesis. PNAS, 104 (44), 17441-17446. https://doi.org/10.1073/pnas.0708559104

Galac, M., \& Lazzaro, B. (2011). Comparative pathology of bacteria in the genus providencia to a natural host, drosophila melanogaster. Microbes Infect, 13, 673-83. https://doi.org/10. 1016/j.micinf.2011.02.005

Galassi, M., Davies, J., Theiler, J., Gough, B., Jungman, G., Alken, P., Booth, M., Rossi, F., \& Ulerich, R. (2009). Gnu scientific library reference manual - third edition (3rd). Network Theory Ltd. www.gnu.org/software/gsl/

Gupta, V., \& Vale, P. F. (2017). Nonlinear disease tolerance curves reveal distinct components of host responses to viral infection. Royal Society Open Science, 4(7). https://doi.org/10. 1098/rsos.170342

Hanson, M., Dostálová, A., Ceroni, C., Poidevin, M., Kondos, S., \& Lemaitre, B. (2019). Synergy and remarkable specificity of antimicrobial peptides in vivo using a systematic knockout approach. eLife, 8, e44341. https://doi.org/10.7554/eLife.44341

Hool, A., Leventhal, G. E., \& Bonhoeffer, S. (2014). Virus-induced target cell activation reconciles set-point viral load heritability and within-host. Epidemics, 7, 36-42. https://doi.org/10. 1016/j.epidem.2014.01.002

Howick, V. M., \& Lazzaro, B. P. (2017). The genetic architecture of defence as resistance to and tolerance of bacterial infection in ji ¿Drosophila melanogasteri/ij. Molecular Ecology, 26, 1533-1546. https://doi.org/10.1111/mec.14017

Huang, J., Lou, Y., Liu, J., Bulet, P., Jiao, R., Hoffmann, J., Liégeois, S., Li, Z., \& Ferrandon, D. (2020). The BaramicinA gene is required at several steps of the host defense against Enterococcus faecalis and Metarhizium robertsii in a septic wound infection model in Drosophila melanogaster. https://doi.org/10.1101/2020.11.23.394809

Jackson, J., Hall, A., Friberg, I., Ralli, C., Lowe, A., Zawadzka, M., Turner, A., Stewart, A., Birtles, R., Paterson, S., Bradley, J., \& Begon, M. (2014). An immunological marker of tolerance 
to infection in wild rodents. PLoS Biology, 12, e1001901. https://doi.org/10.1371/journal. pbio.1001901

Juneja P, L. B. (2009). Providencia sneebia sp. nov. and Providencia burhodogranariea sp. nov. isolated from wild Drosophila melanogaster. Int J Syst Evol Microbiol, 59, 1108-11. https: //doi.org/10.1099/ijs.0.000117-0

King, A. A., Shrestha, S., Harvill, E. T., \& Bjørnstad, O. N. (2009). Evolution of acute infections and the invasion-persistence trade-off. The American Naturalist, 173(4), 446-455. https: //doi.org/10.1086/597217

Kleino, A., \& Silverman, N. (2014). The Drosophila IMD pathway in the activation of the humoral immune response. Developmental and comparative immunology, 42, 25-35. https://doi.org/ https://doi.org/10.1016/j.dci.2013.05.014

Kover, P. X., \& Schaal, B. A. (2002). Genetic variation for disease resistance and tolerance among Arabidopsis thaliana accessions. Proceedings of the National Academy of Sciences of the United States of America, 99(17), 11270-11274. https://doi.org/10.1073/pnas.102288999

Lin, Fulzele, A., Cohen, L. B., Bennett, E. J., \& Wasserman, S. A. (2020). Bombardier enables delivery of short-form bomanins in the drosophila toll response. Frontiers in Immunology, 10, 3040. https://doi.org/10.3389/fimmu.2019.03040

Lin, Y., Parikh, H., \& Y, P. (2018). Stress resistance and lifespan enhanced by downregulation of antimicrobial peptide genes in the IMD pathway. Aging, 10, 622-631. https://doi.org/doi: 10.18632/aging. 101417

Lotka, A. J. et al. (1923). Contribution to the analysis of malaria epidemiology. i. general part. American Journal of Hygiene, 3(Supp). https:// doi.org/10.1093/oxfordjournals.aje. a118963

Louie, A., Song, K. H., Hotson, A., Thomas Tate, A., \& Schneider, D. S. (2016). How many parameters does it take to describe disease tolerance? PLoS Biology, 14(4), e1002435. https: //doi.org/10.1371/journal.pbio.1002435

Mackay, T. F. C., Richards, S., Stone, E. A., Barbadilla, A., Ayroles, J. F., Zhu, D., Casillas, S., Han, Y., Magwire, M. M., Cridland, J. M., Richardson, M. F., Anholt, R. R. H., Barrón, M., Bess, C., Blankenburg, K. P., Carbone, M. A., Castellano, D., Chaboub, L., Duncan, L., .. Gibbs, R. A. (2012). The Drosophila melanogaster genetic reference panel. Nature, 482, 173-178. https://doi.org/doi.org/10.1038/nature10811

Martins, R., Carlos, A. R., Braza, F., Thompson, J. A., Bastos-Amador, P., Ramos, S., \& P, S. M. (2019). Disease tolerance as an inherent component of immunity. Annual Review of Immunology, 37, 405-437. https://doi.org/10.1146/annurev-immunol-042718-041739

Matthews, L., Low, J., Gally, D. L., Pearce, M. C., Mellor, D. J., Heesterbeek, J. A. P., ChaseTopping, M., Naylor, S. W., Shaw, D. J., Reid, S. W. J., Gunn, G. J., \& Woolhouse, M. E. J. (2006). Heterogeneous shedding of Escherichia coli o157 in cattle and its implications for control. Proceedings of the National Academy of Sciences, 103(3), 547-552. https://doi. org/10.1073/pnas.0503776103

Mayer, H., Zaenker, K., \& An Der Heiden, U. (1995). A basic mathematical model of the immune response. Chaos: An Interdisciplinary Journal of Nonlinear Science, 5(1), 155-161. https: //doi.org/10.1063/1.166098

McCarville, J., \& Ayres, J. (2018). Disease tolerance: Concept and mechanisms. Current opinion in immunology, 50, 88-93. https://doi.org/10.1016/j.coi.2017.12.003

Mellors, J. W., Rinaldo, C. R., Gupta, P., White, R. M., Todd, J. A., \& Kingsley, L. A. (1996). Prognosis in HIV-1 infection predicted by the quantity of virus in plasma. Science, 272(5265), 1167-1170. https://doi.org/10.1126/science.272.5265.1167 
Merkling, S. H., Bronkhorst, A. W., Kramer, J. M., Overheul, G. J., Schenck, A., \& Van Rij, R. P. (2015). The epigenetic regulator G9a mediates tolerance to RNA Virus infection in Drosophila. PLoS Pathogens, 11(4), 1-25. https://doi.org/10.1371/journal.ppat.1004692

Petkau, K., Ferguson, M., Guntermann, S., \& Foley, E. (2017). Constitutive immune activity promotes tumorigenesis in Drosophila intestinal progenitor cells. Cell Reports, 20, 1784-1793. https://doi.org/http://dx.doi.org/10.1016/j.celrep.2017.07.078

Pucillo, C., \& Vitale, G. (2020). Crossroads between immune responses and physiological regulation: Metabolic control of resistance versus tolerance against disease. European Journal of Immunology, 50, 484-489. https://doi.org/10.1002/eji.201948159

Pujol, J. M., Eisenberg, J. E., Haas, C. N., \& Koopman, J. S. (2009). The effect of ongoing exposure dynamics in dose response relationships. PLoS Computational Biology, 5(6), e1000399. https://doi.org/10.1371/journal.pcbi.1000399

R Core Team. (2018). R: A language and environment for statistical computing. R Foundation for Statistical Computing. Vienna, Austria. https://www.R-project.org/

Råberg, L., Sim, D., \& Read, A. F. (2007). Disentangling genetic variation for resistance and tolerance to infectious diseases in animals. Science, 318(5851), 812-814. https://doi.org/ $10.1126 /$ science. 1148526

Ramirez-Corona, B., Love, A., Chandrasekaran, S., Prescher, J., \& Wunderlich, Z. (2021). Longitudinal monitoring of individual infection progression in Drosophila melanogaster. bioRxiv. https://doi.org/10.1101/2021.08.17.456698

Read, A. F., Graham, A. L., \& Raberg, L. (2008). Animal defenses against infectious agents: Is damage control more important than pathogen control? PLoS Biology, 6(12), 2638-2641. https://doi.org/10.1371/journal.pbio.1000004

Rousset, F., \& Ferdy, J.-B. (2014). Testing environmental and genetic effects in the presence of spatial autocorrelation. Ecography, 37(8), 781-790. http://dx.doi.org/10.1111/ecog.00566

Shultz, J. M., Espinel, Z., Espinola, M., \& A., R. (2016). Distinguishing epidemiological features of the 2013-2016 West Africa Ebola virus disease outbreak. Disaster Health, 3, 78-88. https: //doi.org/10.1080/21665044.2016.1228326

Soares, M., Teixeira, L., \& Moita, L. (2017). Disease tolerance and immunity in host protection against infection. Nature Reviews Immunology, 17(2), 83. https://doi.org/10.1038/nri. 2016.136

Souto-Maior, C., Sylvestre, G., Dias, F. B. S., Gomes, M. G. M., \& Maciel-de-Freitas, R. (2018). Model-based inference from multiple dose, time course data reveals Wolbachia effects on infection profiles of type 1 dengue virus in Aedes aegypti. PLoS Neglected Tropical Diseases, 12(3), e0006339. https://doi.org/10.1371/journal.pntd.0006339

Stoecklein, V. M., Osuka, A., \& Lederer, J. A. (2012). Trauma equals danger-damage control by the immune system. Journal of Leukocyte Biology, 92(3), 539-551. https://doi.org/10. $1189 /$ jlb.0212072

Therneau, T. M. (2020). Coxme: Mixed effects cox models [R package version 2.2-16]. https:// CRAN.R-project.org/package $=$ coxme

Troha, K., Im, J. H., Revah, J., Lazzaro, B. P., \& Buchon, N. (2018). Comparative transcriptomics reveals $C r e b A$ as a novel regulator of infection tolerance in D. melanogaster. PLoS Pathogens, 14(2), 1-34. https://doi.org/10.1371/journal.ppat.1006847

Unckless, R. L., Howick, V. M., \& Lazzaro, B. P. (2016). Convergent balancing selection on an antimicrobial peptide in Drosophila. Current Biology, 26(2), 257-262. https://doi.org/10. 1016/j.cub.2015.11.063 
van Kampen, N. (1985). Elimination of fast variables. Physics Reports, 124, 69-160. https://doi. org/10.1016/0370-1573(85)90002-X

van Leeuwen, A., Budischak, S. A., Graham, A. L., \& Cressler, C. E. (2019). Parasite resource manipulation drives bimodal variation in infection duration. Proc. R. Soc. B, 286, 20190456. https://doi.org/https://doi.org/10.1098/rspb.2019.0456

Virgin, H. W., Wherry, E. J., \& Ahmed, R. (2009). Redefining chronic viral infection. Cell, 138(1), 30-50. https://doi.org/10.1016/j.cell.2009.06.036

Volterra, V. (1928). Variations and fluctuations of the number of individuals in animal species living together. ICES Journal of Marine Science, 3(1), 3-51. https://doi.org/doi.org/10. 1093/icesjms/3.1.3 


\section{S1 The equilibrium states of the infection}

\section{S1.1 High constitutive immunity is required to cure infections, but may kill the host}

Clearance is the equilibrium characterized by $x=0$. Let $\tilde{y}_{h}$ and $\tilde{z}_{h}$ be the equilibrium levels of defense and damage for this equilibrium (with $\tilde{y}_{h}$ the homeostatic level of defense). From equations 1 , we know that $\tilde{z}_{h}=\tilde{y}_{h} \eta / \xi$ so that $\tilde{y}_{h}$ is a solution of $\gamma G\left(\tilde{y}_{h}, \eta / \xi \tilde{y}_{h}\right)=\varphi \tilde{y}_{h}$, which yields

$$
-\frac{\gamma}{\varphi}+\tilde{y}_{h}+\tilde{y}_{h}^{k+1}+\psi\left(\frac{\eta}{\xi} \tilde{y}_{h}\right)^{l+1}=0 .
$$

The left term of equation (S1-1) is a strictly increasing function of $\tilde{y}_{h}$, which has exactly one positive root because $-\gamma / \varphi \leq 0$. From equation (S1-1), it is also easy to see that this root increases with $\gamma$.

A necessary condition for clearance to be stable is that $d x / d t\left(0, \tilde{y}_{h}, \tilde{z}_{h}\right)<0$ which requires that $\tilde{y}_{h}>1 / \delta$. From this and the previous result, we conclude that clearance is stable if and only if $\gamma>\gamma_{c}$ with

$$
\begin{aligned}
\gamma_{c} & =\frac{\varphi}{\delta} \frac{1}{G(1 / \delta, \eta /(\delta \xi))}, \\
& =\frac{\varphi}{\delta}\left(1+\left(\frac{1}{\delta}\right)^{k}+\psi\left(\frac{\eta}{\xi \delta}\right)^{l}\right) .
\end{aligned}
$$

Increasing $\gamma$ raises the level of defense $y$, even in the absence of infection (as figure 3B illustrates); but it also add to damage $z$, because immune defense have negative side effects when $\eta>0$ (see figure 3C). If constitutive defense are over-expressed, these negative effects could even kill the host in the absence of any infection. This condition is met when $\tilde{z}_{h}>$ $z_{d} \Leftrightarrow \gamma>\gamma_{s}$ with

$$
\gamma_{s}=\varphi \frac{\xi z_{d}}{\eta}\left(1+\left(\frac{\xi z_{d}}{\eta}\right)^{k}+\psi z_{d}^{l}\right)
$$

\section{S1.2 Bistability requires that damage hinders defense production}

Let $\tilde{x}, \tilde{y}$ and $\tilde{z}$ be the state variables at equilibrium. Let $\bar{y}(x)$ and $\bar{z}(x)$ be the $y$ and $z$ values such that $\frac{d x}{d t}(x, \bar{y}(x), \bar{z}(x))=\frac{d z}{d t}(x, \bar{y}(x), \bar{z}(x))=0$. From equation (1), it is easily shown that

$$
\left\{\begin{array}{l}
\bar{y}(x)=(1-x) / \delta \\
\bar{z}(x)=(x(\omega-\eta / \delta)+\eta / \delta) / \xi
\end{array}\right.
$$

with $\tilde{y}=\bar{y}(\tilde{x})$ and $\tilde{z}=\bar{z}(\tilde{x})$. The equilibrium load $\tilde{x}$ can be found by solving $d y / d t(x, \bar{y}(x), \bar{z}(x))=$ 0 , which can be written as

$$
H(\tilde{x})=F(\tilde{x}) G(\bar{y}(\tilde{x}), \bar{z}(\tilde{x}))-\varphi \bar{y}(\tilde{x})=0 .
$$




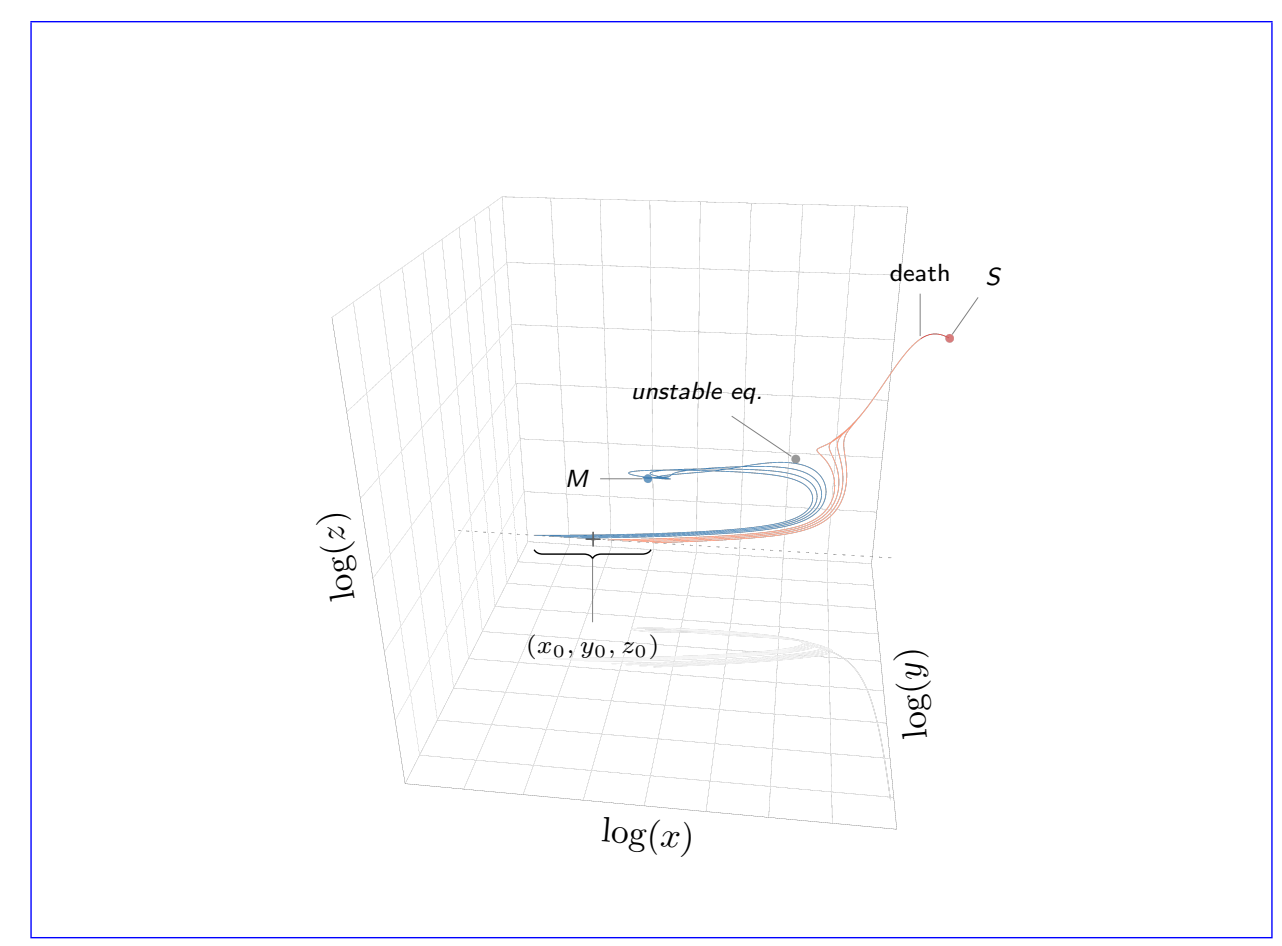

Figure S1-1: The dynamics of the system in a bistable configuration.

The system is therefore bistable only if $H$ has at least two roots in $[0,1]$, which implies that $H^{\prime}$ has at least one root in the same range. We obtain

$$
\begin{aligned}
H^{\prime}(x)= & F^{\prime}(x) G(\bar{y}(x), \bar{z}(x)) \\
& \quad+F(x)\left(\dot{\bar{y}}(x) \frac{\partial G}{\partial y}(\bar{y}(x), \bar{z}(x))+\dot{\bar{z}}(x) \frac{\partial G}{\partial z}(\bar{y}(x), \bar{z}(x))\right)-\varphi \dot{\bar{y}}(x) .
\end{aligned}
$$

with $\dot{\bar{y}}=d \bar{y} / d x=-1 / \delta$ and $\dot{\bar{z}}=d \bar{z} / d x=(\omega-\eta / \delta) / \xi . F^{\prime}$ is positive under the assumption that $u \geq v$ and all derivatives of $G$ are negative. If $\psi=0, \partial G / \partial z=0$ and all remaining terms in equation (S1-6) are positive. $H^{\prime}$ therefore never cancels and $H$ must have a single root: the system cannot be bistable. If, conversely, $\psi>0$ and if $\omega>\eta / \delta$, then $\dot{\bar{z}} \partial G / \partial z>0$ and $H$ can have more than one root. $\psi>0$ and $\theta=\omega-\eta / \delta>0$ are therefore necessary conditions for the system to be bistable.

\section{S2 Load and damage at equilibrium}

\section{S2.1 Co-variations between load and damage at equilibrium}

We took advantage of the implicit function theorem to gain information on how $\tilde{x}$ and $\tilde{z}$ covary when parameters are changed. Let us define $H_{x}, H_{y}$ and $H_{z}$ as

$$
\left\{\begin{array}{l}
H_{x}(x, y, z)=d x / d t \\
H_{y}(x, y, z)=d y / d t \\
H_{z}(x, y, z)=d z / d t
\end{array}\right.
$$


so that $H_{x}(\tilde{x}, \tilde{y}, \tilde{z})=H_{y}(\tilde{x}, \tilde{y}, \tilde{z})=H_{z}(\tilde{x}, \tilde{y}, \tilde{z})=0$. Assuming $\tilde{x}>0$ and $\tilde{z}>0, H_{x}, H_{y}$ and $H_{z}$ are all smooth. The Jacobian matrix $\mathrm{J}$ of our system is defined by the partial derivatives of functions $H_{x}, H_{y}$ and $H_{z}$ which, taken at the equilibrium point, yields

$$
J(\tilde{x}, \tilde{y}, \tilde{z})=\left(\begin{array}{ccc}
-\tilde{x} & -\delta \tilde{x} & 0 \\
F^{\prime}(\tilde{x}) G(\tilde{y}, \tilde{z}) & F(\tilde{x}) \frac{\partial G}{\partial y}(\tilde{y}, \tilde{z})-\varphi & F(\tilde{x}) \frac{\partial G}{\partial z}(\tilde{y}, \tilde{z}) \\
\omega & \eta & -\xi
\end{array}\right)
$$

of which determinant is

$$
|J(\tilde{x}, \tilde{y}, \tilde{z})|=-\xi \delta \tilde{x} H^{\prime}(\tilde{x}) .
$$

Assuming that $\tilde{x}>0,|J(\tilde{x}, \tilde{y}, \tilde{z})|$ will be zero when $H^{\prime}(\tilde{x})=0$. This condition is met only in the limit case which corresponds to the boundary of the bistable parameter region (see figure 4). In all other situations, therefore, $|J(\tilde{x}, \tilde{y}, \tilde{z})| \neq 0$ and the implicit function theorem applies in the neighbourhood of $(\tilde{x}, \tilde{y}, \tilde{z})$. Each equilibrium variable $\tilde{x}, \tilde{y}$ and $\tilde{z}$ can then be written as a unique function of parameters which partial derivatives might be obtained by implicit differentiation.

\section{S2.1.1 The effect of parameters involved in response modulation}

Let us first consider the effects of variation in $\gamma$, the reasoning applying in fact to any parameter which is not directly involved in $H_{x}$ and $H_{z}$. We have

$$
\left\{\begin{array}{l}
\frac{\partial H_{x}}{\partial \gamma}=0=(1-\tilde{x}-\delta \tilde{y}) \frac{\partial \tilde{x}}{\partial \gamma}-\tilde{x}\left(\frac{\partial \tilde{x}}{\partial \gamma}+\delta \frac{\partial \tilde{y}}{\partial \gamma}\right), \\
\frac{\partial H_{z}}{\partial \gamma}=0=\omega \frac{\partial \tilde{x}}{\partial \gamma}+\eta \frac{\partial \tilde{y}}{\partial \gamma}-\xi \frac{\partial \tilde{z}}{\partial \gamma} .
\end{array}\right.
$$

Let us now assume that $\tilde{x}>0$, i.e. the infection has not been cleared at equilibrium. We obtain

$$
\left\{\begin{array}{l}
\frac{\partial \tilde{x}}{\partial \gamma}+\delta \frac{\partial \tilde{y}}{\partial \gamma}=0 \\
\omega \frac{\partial \tilde{x}}{\partial \gamma}+\eta \frac{\partial \tilde{y}}{\partial \gamma}-\xi \frac{\partial \tilde{z}}{\partial \gamma}=0
\end{array}\right.
$$

which, by eliminating $\frac{\partial \tilde{y}}{\partial \gamma}$, yields

$$
\frac{\partial \tilde{z}}{\partial \gamma}=\frac{\left(\omega-\frac{\eta}{\delta}\right)}{\xi} \frac{\partial \tilde{x}}{\partial \gamma}
$$

As long as $\theta=\omega-\frac{\eta}{\delta}>0$, damage therefore increases with load. If ever $\theta<0$, increasing defense (e.g. by increasing $\gamma$ ) will decrease load but increase damage. In these circumstances, the secondary effects associated to greater defense production exceeds the benefit of better control over pathogen proliferation.

\section{S2.1.2 The effect of defense efficiency}

The same type of calculation has been applied to other parameters, including $\delta$. This time, we obtain

$$
\frac{\partial \tilde{x}}{\partial \delta}+\tilde{y}+\delta \frac{\partial \tilde{y}}{\partial \delta}=0 \Rightarrow \frac{\partial \tilde{y}}{\partial \delta}=-\frac{1}{\delta} \frac{\partial \tilde{x}}{\partial \delta}-\frac{\tilde{y}}{\delta}
$$


Compared to the effect of changes in $\gamma$ (equation (S2-11)), equation (S2-13) shows that changes in $\delta$ will not necessarily produced variations in $\tilde{y}$ which are proportional to that in $\tilde{x}$. Writing $\partial H_{z} / \partial \delta$ and eliminating $\frac{\partial \tilde{y}}{\partial \delta}$, we now obtain

$$
\frac{\partial \tilde{z}}{\partial \delta}=\frac{\left(\omega-\frac{\eta}{\delta}\right)}{\xi} \frac{\partial \tilde{x}}{\partial \delta}-\frac{\tilde{y}}{\xi \delta} .
$$

As in equation (S2-12), $\partial \tilde{x} / \partial \delta$ and $\partial \tilde{z} / \partial \delta$ might have opposite signs. But this time, the sign difference cannot be predicted from the sole sign of $\theta$. If $\theta>0$, any decrease in $\tilde{x}$ following an increase in $\delta$ will indeed be accompanied by a decrease in $\tilde{z}$; but the same types of variation may occur when $\theta<0$, provided that $\tilde{y} / \delta$ is high enough.

\section{S2.1.3 The effect of parameters involved in damage dynamics}

Following the same line of reasoning, we can describe how changes in $\omega, \eta$ and $\xi$ impact load and damage at equilibrium:

$$
\begin{aligned}
& \frac{\partial \tilde{z}}{\partial \omega}=\frac{\left(\omega-\frac{\eta}{\delta}\right)}{\xi} \frac{\partial \tilde{x}}{\partial \omega}+\frac{\tilde{x}}{\xi}, \\
& \frac{\partial \tilde{z}}{\partial \eta}=\frac{\left(\omega-\frac{\eta}{\delta}\right)}{\xi} \frac{\partial \tilde{x}}{\partial \eta}+\frac{\tilde{y}}{\xi} \\
& \frac{\partial \tilde{z}}{\partial \xi}=\frac{\left(\omega-\frac{\eta}{\delta}\right)}{\xi} \frac{\partial \tilde{x}}{\partial \xi}-\frac{\tilde{z}}{\xi} .
\end{aligned}
$$

In these three expressions, as in equation (S2-14), the change in $\tilde{z}$ cannot be determined solely from that of $\tilde{x}$ and from $\theta=\omega-\frac{\eta}{\delta}$. In fact, because we are here varying parameters which directly control damage dynamics, right hand terms in equations (S2-15), (S2-16) and (S2-17) all include a term which quantifies this direct effect $(\tilde{x} / \xi, \tilde{y} / \xi$ and $-\tilde{z} / \xi$ respectively). The other term rather measures damage variations which are indirect consequences of load variations.

\section{S2.2 Variations in equilibrium load when parameters vary}

We shall now write the partial derivatives of $H(\tilde{x})$ (viewed as a function of the parameters) to obtain derivatives of $\tilde{x}$ relative to each parameter. Starting with $\gamma$, we obtain

$$
\begin{aligned}
\frac{\partial H(\tilde{x})}{\partial \gamma}=( & \left.+\frac{\partial \tilde{x}}{\partial \gamma} F^{\prime}(\tilde{x})\right) G(\tilde{y}, \tilde{z}) \\
& +F(\tilde{x})\left(\frac{\partial \tilde{y}}{\partial \gamma} \frac{\partial G}{\partial y}(\tilde{y}, \tilde{z})+\frac{\partial \tilde{z}}{\partial \gamma} \frac{\partial G}{\partial z}(\tilde{y}, \tilde{z})\right)-\varphi \frac{\partial \tilde{y}}{\partial \gamma}=0
\end{aligned}
$$

The right hand term of equation (S2-18) simplifies to $G(\tilde{y}, \tilde{z})+\frac{\partial \tilde{x}}{\partial \gamma} H^{\prime}(\tilde{x})$ which therefore yields

$$
\frac{\partial \tilde{x}}{\partial \gamma}=-\frac{G(\tilde{y}, \tilde{z})}{H^{\prime}(\tilde{x})}
$$


with $H^{\prime}$ the derivative of $H$ given by equation (S1-6). Assuming that $(\tilde{x}, \tilde{y}, \tilde{z})$ is a stable equilibrium, we know that $|J(\tilde{x}, \tilde{y}, \tilde{z})|<0$. Using equation (S2-9), we can conclude that $H^{\prime}(\tilde{x})$ is positive, and equation (S2-19) therefore predicts that any increase in $\gamma$ will induce a decrease in $\tilde{x}$.

Let us now consider the case of the unstable equilibria with $\tilde{x}>0$, which exists only when the system is bistable. Using the Routh-Hurwitz criteria for $3 \times 3$ matrices, we can state that $J(\tilde{x}, \tilde{y}, \tilde{z})$ is stable if the three conditions $\operatorname{tr} J(\tilde{x}, \tilde{y}, \tilde{z})<0,|J|<0$ and $\operatorname{tr} J(\tilde{x}, \tilde{y}, \tilde{z}) a<$ $|J(\tilde{x}, \tilde{y}, \tilde{z})|$ are all satisfied, with $\operatorname{tr} J(\tilde{x}, \tilde{y}, \tilde{z})$ the trace and $a$ the sum of all principal $2 \times 2$ principal minors of $J(\tilde{x}, \tilde{y}, \tilde{z})$. From equation (S2-8), it is clear that the first condition is always met. It can also easily be demonstrated that, the system being bistable which requires that $\theta>0, a>\tilde{x} \delta H^{\prime}(\tilde{x})$. From this, it can be shown that the third condition of Routh-Hurwitz criteria is also always satisfied. We can therefore conclude that if $(\tilde{x}, \tilde{y}, \tilde{z})$ is unstable, $|J(\tilde{x}, \tilde{y}, \tilde{z})|>0$ and therefore $H^{\prime}(\tilde{x})<0$. This result yields the simple conclusion that, at the unstable equilibria of a bistable system, any increase in $\gamma$ will make $\tilde{x}$ decrease.

The same sort of calculation can be performed for each parameter. Table S2-1 summarizes these results, with the sign of derivatives being given for both stable and unstable equilibria. Figure S2-2 gives a numerical illustration of these computations.

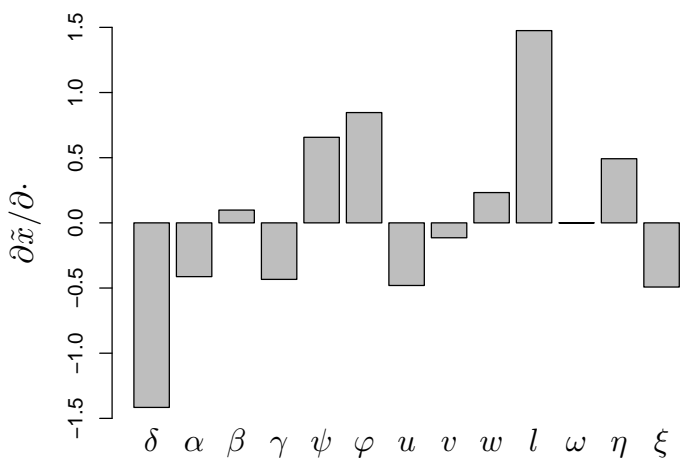

Figure S2-2: Elasticity analysis of equilibrium load. Parameters are here as in Figure 2 and the derivatives of $\log$ equilibrium load $\tilde{x}$ relative to log parameters, obtained from table S2-1, are computed for the low load equilibrium. For this equilibrium, $\tilde{x}$ is the load at which the chronic infection stabilizes and can therefore be considered as a SPPL. The parameters with greatest influence on SPPL are first the efficiency of defense $(\delta)$ and second the parameter which shapes the impact of damage on defense production $(l)$. 


\begin{tabular}{|c|c|c|c|c|}
\hline & $\partial \tilde{x} / \partial$ & & st.eq. & unst.eq. \\
\hline$\delta$ & $\begin{array}{r}-\frac{\varphi \tilde{y}}{\delta}\left(k \tilde{y}^{k}+\frac{\psi \eta}{\xi} l \tilde{y} \tilde{z}^{l-1}\right) \times \\
-\frac{\varphi}{\delta} \tilde{y} \times\end{array}$ & $\begin{array}{l}\left.\frac{G(\tilde{y}, \tilde{z}}{H^{\prime}(\tilde{x})}\right) \\
\frac{1}{H^{\prime}(\tilde{x})}\end{array}$ & $<0$ & $>0$ \\
\hline$\alpha$ & $-\frac{\tilde{x}^{u}}{1+\beta \tilde{x}^{v}} \times$ & $\frac{G(\tilde{y}, \tilde{z})}{H^{\prime}(\tilde{x})}$ & $<0$ & $>0$ \\
\hline$\beta$ & $\alpha \frac{\tilde{x}^{u+v}}{\left(1+\beta \tilde{x}^{v}\right)^{2}} \times$ & $\frac{G(\tilde{y}, \tilde{z})}{H^{\prime}(\tilde{x})}$ & $>0$ & $<0$ \\
\hline$\gamma$ & & $\frac{G(y, z)}{H^{\prime}(\tilde{x})}$ & $<0$ & $>0$ \\
\hline$\psi$ & $\varphi \tilde{y} \tilde{z}^{l} \times$ & $\frac{G(y, z)}{H_{1}^{\prime}(\tilde{x})}$ & $>0$ & $<0$ \\
\hline$\varphi$ & $\tilde{y} \times$ & & $>0$ & $<0$ \\
\hline$u$ & $-\log (\tilde{x})(F(\tilde{x})-\gamma) \times$ & $\frac{G(\tilde{y}, \tilde{z})}{H^{\prime}(\tilde{x})}$ & $>0$ & $<0$ \\
\hline$v$ & $\log (\tilde{x}) \frac{\alpha \beta \tilde{x}^{u+v}}{(1+\beta \tilde{x})^{2}} \times$ & $\frac{G(\tilde{y}, \tilde{z})}{H^{\prime}(\tilde{x})}$ & $<0$ & $>0$ \\
\hline$k$ & $\log (\tilde{y}) \varphi \tilde{y}^{k+1} \times$ & $\frac{G(\tilde{y}, \tilde{z})}{H^{\prime}(\tilde{x})}$ & $>0$ if $\tilde{y}>1$ & $<0$ if $\tilde{y}>1$ \\
\hline$l$ & $\log (\tilde{z}) \psi \varphi \tilde{y} \tilde{z}^{l} \times$ & $\frac{G(\tilde{y}, \tilde{z})}{H^{\prime}(\tilde{x})}$ & $>0$ if $\tilde{z}>1$ & $<0$ if $\tilde{z}>1$ \\
\hline$\omega$ & $\frac{\varphi \psi l}{\xi} \tilde{x} \tilde{y} \tilde{z}^{l-1} \times$ & $\frac{G(\tilde{y}, \tilde{z})}{H^{\prime}(\tilde{x})}$ & $>0$ & $<0$ \\
\hline$\eta$ & $\frac{\varphi \psi l}{\xi} \tilde{y}^{2} \tilde{z}^{l-1} \times$ & $\frac{G(\tilde{y}, \tilde{z})}{H^{\prime}(\tilde{x})}$ & $>0$ & $<0$ \\
\hline$\xi$ & $-\frac{\varphi \psi l}{\xi} \tilde{y} \tilde{z}^{l} \times$ & $\frac{G(\tilde{y}, \tilde{z})}{H^{\prime}(\tilde{x})}$ & $<0$ & $>0$ \\
\hline
\end{tabular}

Table S2-1: Derivatives of equilibrium load with respect to model parameters. For each parameter, the derivative is given together with its sign for stable and unstable equilibria. 


\section{S3 The Set Point Pathogen Load (SPPL)}

\section{S3.1 Definition: stable vs. transient SPPL}

The SPPL can be defined as a sub-lethal load which stabilizes for a significant time during the infection. This corresponds well to what has been historically measured on HIV infections, but it remains vague as long as we do not specify what a "significant time" is. In a mathematical model of infection, the SPPL could correspond to a stable equilibrium load, at which the infection stabilizes permanently. Its relation to parameters is then described by the analysis presented in S2-1.

But we shall see in the next section that the SPPL can also corresponds to non-equilibrium situations, where the progression of the infection slows down temporarily only. We must therefore distinguish stable from transient SPPL, which both characterize chronic infections but have, as we shall see, very different properties.

\section{S3.2 Bistability can make the SPPL transient}

A transient SPPL is a load which stabilizes for a long but finite period of time. This situation somehow resembles that of a chronic infection, but control is only transient and the infection will eventually enter a final phase where it reaches high loads and finally kills the host.

The progression of the infection slows down when a transient SPPL is approached because the trajectory evolves in the neighbourhood of an equilibrium point; the fact that the infection eventually moves away from this point demonstrates that this equilibrium is unstable. Therefore, the SPPL cannot be transient unless an unstable equilibrium exists with $\tilde{x}>0$, which in turns requires that the system is bistable.

In such conditions, the pathogen load typically first peaks, then decreases until it reaches a plateau (a local minimum) and finally increases again while the disease proceeds towards its final phase. We computed the SPPL is such situations as the load at the local minimum. Computing the duration of chronicity is not as straightforward. We considered that control starts when load peaks, because it corresponds to the time at which immunity manages to curb bacterial proliferation. We further considered that control ends when load, after having decreased to the SPPL, reaches again the peak load. We estimated the duration of chronicity as the time that elapses between these two moments.

In most conditions, increasing $x_{0}$, decreasing $y_{0}$ or increasing $z_{0}$ increases the SPPL and shortens chronicity (fig. S3-1). In summary anything that strengthens immunity does lower the SPPL and, most of the time, lengthen chronicity. Exceptions to this rule exist, though: figure S3-1 indeed illustrates cases (e.g. when $\psi=0.5$ ) where the duration of chronicity decreases when $y_{0}$ is increased. It should be noted, however, that this unexpected result may originate from the way we have defined the duration of control. We chose to start the period of transient chronicity at the peak that precedes the SPPL but other methods could be imagined, and it may be that some of the results we have obtained on this quantity depend on how it is precisely defined.

When the SPPL is transient, both its value and the duration of control vary with initial conditions, because they both depend on how close the trajectory will get from the unstable equilibrium. When stable, the SPPL corresponds to a stable equilibrium. It therefore lasts 


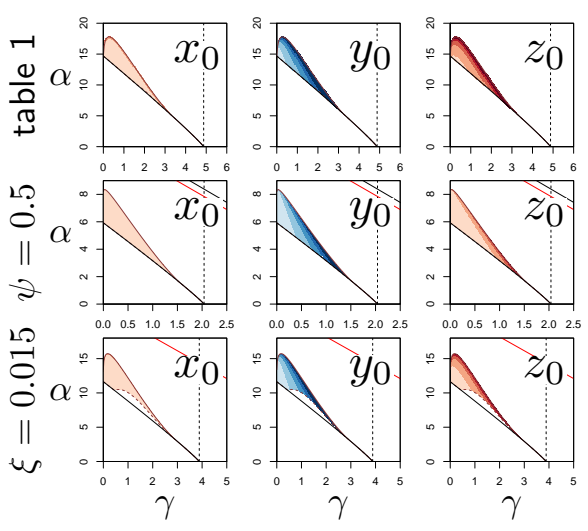

partial derivative of log SPPL relative to log initial conditions

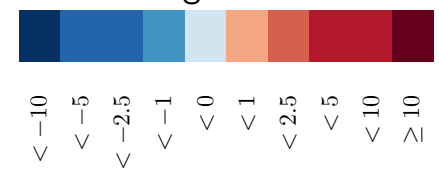

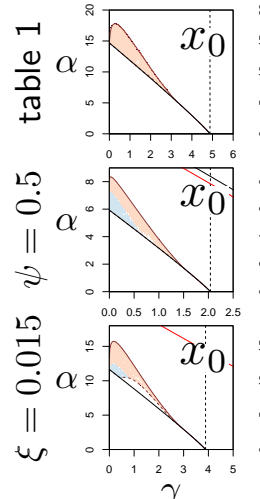
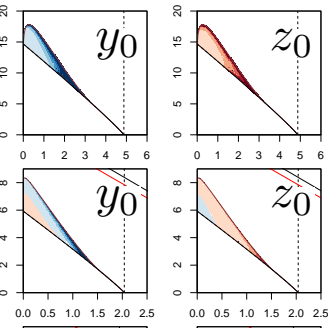

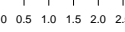
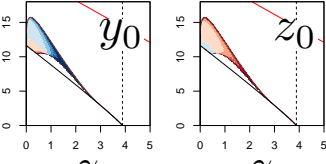

partial derivative of log duration of chronicity relative to log initial conditions

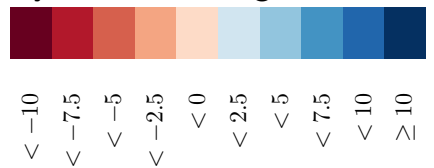

Figure S3-1: The influence of initial conditions on transient SPPL. Left panel: each graph represents the partial derivative of log-SPPL relative to log-transformed inoculum size $\left(\log \left(x_{0}\right)\right.$, first column), pre-infection level of defense $\left(\log \left(y_{0}\right)\right.$, second column) and pre-infection level of damage $\left(\log \left(z_{0}\right)\right.$, third column) with $x_{0}=10^{-6}$ and $y_{0}$ and $z_{0}$ set to the homeostatic state. In the first row, parameters are set as in figure 2 , while $\psi=0.5$ in the second row, and $\xi=0.015$ in the third. The red envelop delimits the parameter region inside which the SPPL is transient. The case $u=0.75$, which we analyzed in figure 4 , is not presented here as the SPPL cannot be transient in this condition. The blue color indicates better control of the infection, i.e. decreased SPPL, while red means increased SPPL. In each case, anything that weakens the host (higher $x_{0}$, lower $y_{0}$ or higher $z_{0}$ ) lowers the SPPL. The analysis also indicates that sensitivity to variations in $y_{0}$ and $z_{0}$ increase with both $\alpha$ and $\gamma$. Right panel: This analysis follows the same logic but the partial derivatives of the log of the duration of chronicity are computed. The blue color indicates again better control, i.e. longer chronicity. In most cases, anything that weakens the host shortens control. But conditions exist where this is not true (e.g. in second and third rows when $\gamma$ and $\alpha$ are low). Contrary to what is expected, chronicity may therefore last longer with increased SPPL when immunity is weak. 
forever (which in more realistic terms means that the host will die from something else than the infection) and does not depend on initial conditions. This provides a method to experimentally distinguish the two situations: if the SPPL increases with the dose of pathogen a host is infected by, it must be transient. This could be used as an indirect way to demonstrate first that the system constituted by the interacting host and pathogens is bistable, and second that damage caused by the infection hinders the production of immune defense (see section S1.2).

\section{S3.3 A sensibility analysis of transient SPPL}

We propose that anything that strengthens the host immunity decreases the SPPL and, in most situations, lengthens the duration of control. We explored this rule in more details by running a elasticity analysis of the SPPL and the duration of chronicity with $x_{0}=10^{-6}$ and $y_{0}$ and $z_{0}$ set at their homeostatic state (see fig. S3-2 and S3-3). For each parameter, we numerically computed the derivative of the SPPL using a five point approximation. In the case of parameter $u$, we computed right derivatives, so that the condition $u>v$ holds in our computation. Similarly, we did not compute derivatives with respect to parameter $v$ alone, but rather varied both $u$ and $v$ at the same time, keeping $u=v$, so that $F$ always increases with $x$. Overall, we found that increasing the production of defense (by increasing $\gamma$ or $\alpha$ ) and making those defense more efficient (by increasing $\delta$ ) lower the SPPL. Increasing the rate at which damage is repaired (by increasing $\xi$ ) does the same (fig. S3-2).

What determines variation in the duration control is more complex. For example, when $\psi=0.5$ parameter combinations exists for which the duration of control increases when immunity causes more damage (i.e. when $\eta$ increases, see fig. S3-3). This is reminiscent to the situation we discussed in the previous section, and again we must warn that different ways of calculating duration of control are possible and that this paradoxical result might come from the method we have chosen. It should be noted however that the parameter range over which the duration of control is not negatively related to the value of SPPL is extremely limited. 

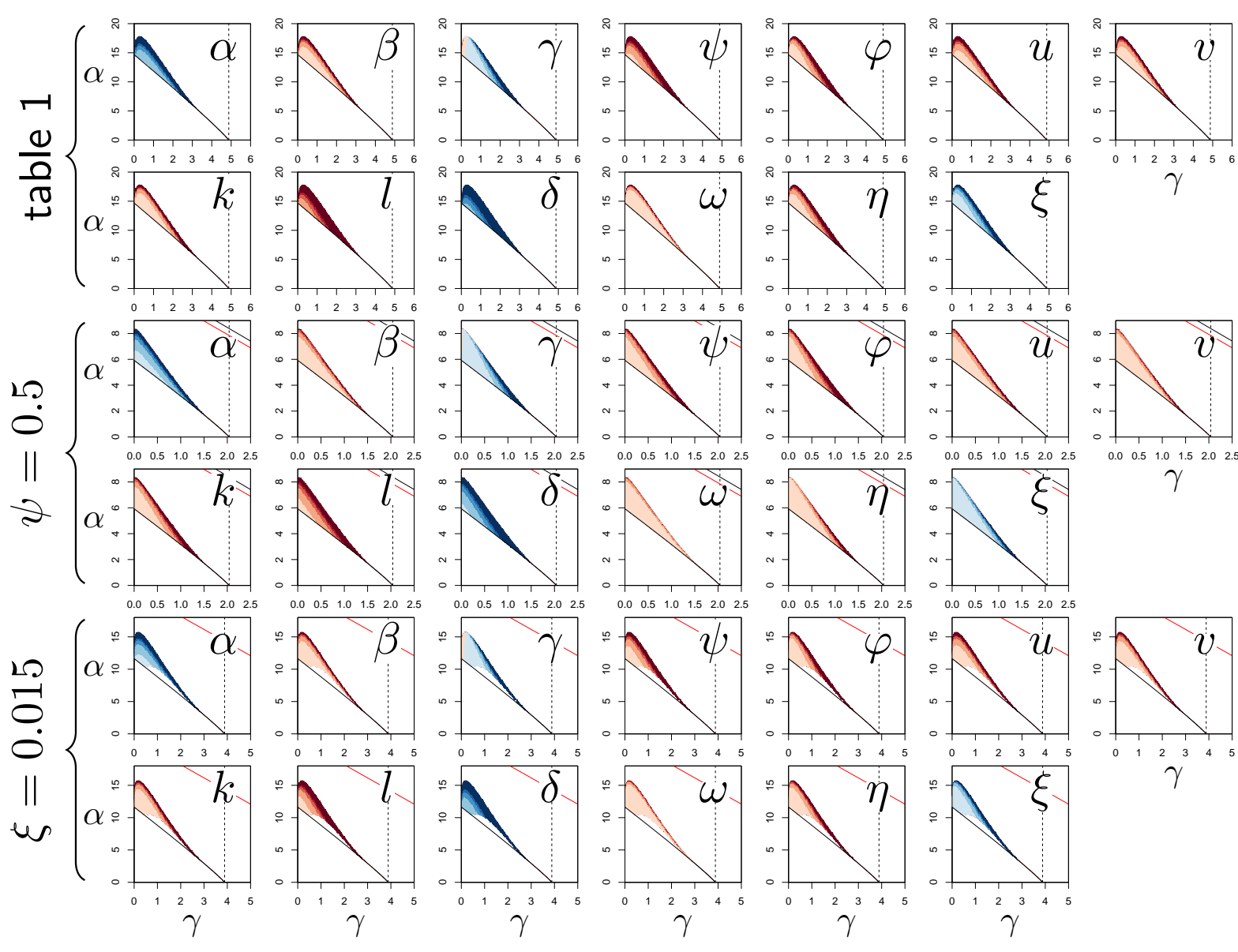

$\gamma$
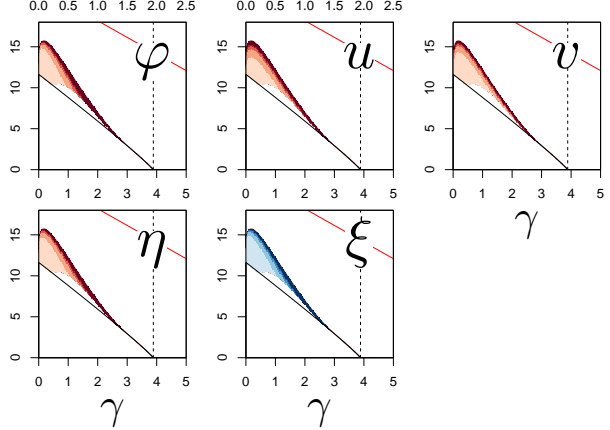

partial derivative of $\log$ SPPL relative to log parameters

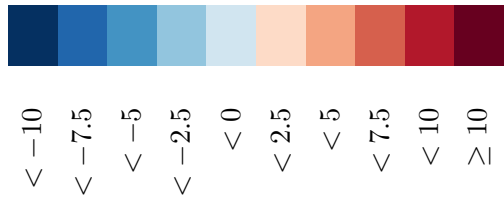

Figure S3-2: Elasticity analysis of SPPL in transient chronicity according to parameters. Each graph represents the partial derivative of log SPPL with respect to one (log-transformed) parameter, as a function of $\alpha$ and $\gamma$. Blue color corresponds to negative derivatives (i.e. increasing the parameter lowers the SPPL) while red indicates positive derivatives (i.e. increasing the parameter increases the SPPL). The pairs or rows correspond to the three parameter sets studied in figure S3-1. Derivatives with respect to $z_{d}$ have been omitted in this analysis as, by definition, $z_{d}$ has no influence on SPPL. 

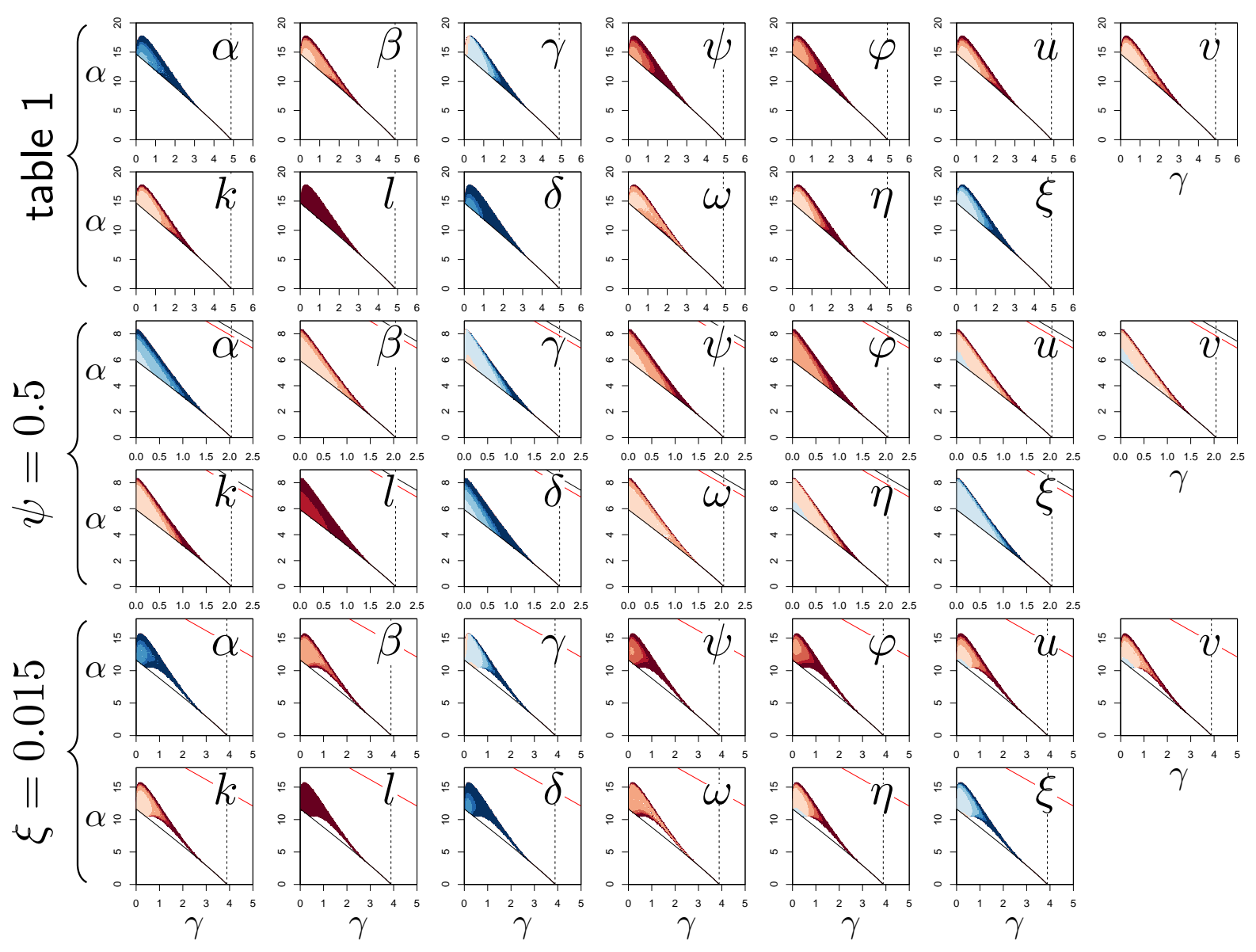

partial derivative of log duration of chronicity relative to log parameters

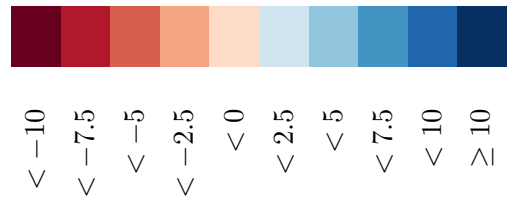

Figure S3-3: Elasticity analysis of the duration of transient chronicity according to parameters. Each graph represents the partial derivative of the log duration of chronicity with respect to one (log-transformed) parameter, as a function of $\alpha$ and $\gamma$. Blue color corresponds to positive derivatives (i.e. increasing the parameter lengthen chronicity) while red indicates negative derivatives (i.e. increasing the parameter shortens chronicity). The three pairs of rows correspond to the three parameter sets studied in figures S3-1. Derivatives with respect to $z_{d}$ have been omitted in this analysis as, by definition, $z_{d}$ has no influence on SPPL. 


\section{S4 The Pathogen Load Upon Death (PLUD)}

\section{S4.1 Definition}

The PLUD is the load $x_{d}$ at which a host dies. It is therefore the $x$ coordinate of the unique point where a trajectory first intersects the plane defined by $z=z_{d}$, with $z_{d}$ the amount of damage the host can sustain.

\section{S4.2 Why is the PLUD almost independent from initial condi- tions?}

In principle, the exact form of a trajectory depends on its starting point. The PLUD should therefore also vary with initial conditions. But trajectories tend to merge when approaching the high load equilibrium, as can be seen in figure S1-1; if this occurs before $z$ has exceeded $z_{d}$, different infections will kill their host at similar loads, even though they do so at very different times.

This particular behaviour comes from the fact that in infections which enter their final stage, just before the host dies, accumulating damage makes defense decrease. This implies that $\frac{d y}{d t}<0$ and thus $0<f(x) g(y, z) / y<\varphi$. Assuming that $\varphi$ is small and $y$ moderate, we can consider that $f(x) g(y, z)$ is also small so that $y$ is a slow variable compared to $x$. Because $x$ depends on $y$ but not on $z$, it should therefore rapidly get close to $\frac{d x}{d t}=0$.

Said differently, if defense decay is a slow process, pathogens should rapidly reach the highest possible load allowed by the current amount of defense $\left(\frac{d x}{d t} \gtrsim 0 \Rightarrow x_{t} \lesssim 1-\delta y_{t}\right)$. The dynamics of $x$ will then be "enslaved" by that of $y$ (van Kampen, 1985) and load will increase slowly while $y$ gradually diminishes under the effect of accumulating damage. Because of this very particular dynamics, the way $x_{t}$ relates to $z_{t}$ will almost be the same for all infections that enter their final phase, which in the end renders the PLUD almost independent from the conditions that prevailed at the onset of the infection.

Figure $5 \mathrm{C}$ gives a simple illustration of this result. Figure S4-1 provides a more complete elasticity analysis, where the impact of variations in $x_{0}, y_{0}$ and $z_{0}$ are systematically explored following the same logic as in figure S3-1. The comparison with figure S3-1, where the SPPL is analyzed, demonstrates that variations in initial conditions have a much weaker impact on the PLUD than on the SPPL (derivatives relative to initial conditions span from -0.1 to 0.1 for the PLUD, see fig. S4-1, while they span from -10 to 10 for the SPPL, and fig. S3-1).

\section{S4.3 A lower bound for the PLUD}

In conditions where $z$ also evolves faster than $y$ during the final stage of the disease, trajectories leading to host's death should rapidly approach the intersect of the two isocline planes defined by $d x / d t=0$ and $d z / d t=0$. Building on this, we assume that points on the trajectory should then satisfy $y_{t}=\bar{y}\left(x_{t}\right)$ (i.e. $d x / d t=H_{x}(x, y)=0$ ) and $x_{t}=\bar{x}\left(z_{t}\right)=\left(\xi z_{t}-\eta / \delta\right) /(\omega-\eta / \delta)$ (i.e. $\left.d z / d t=H_{z}(x, y, z)=0\right)$. From there, we obtain that the host should die at a load close to $\bar{x}\left(z_{d}\right)=\left(\xi z_{d}-\eta / \delta\right) /(\omega-\eta / \delta)$. Note that if the high load equilibrium satisfies $\tilde{z}=z_{d}$, the PLUD is then exactly $x_{d}=\bar{x}\left(z_{d}\right)$. 

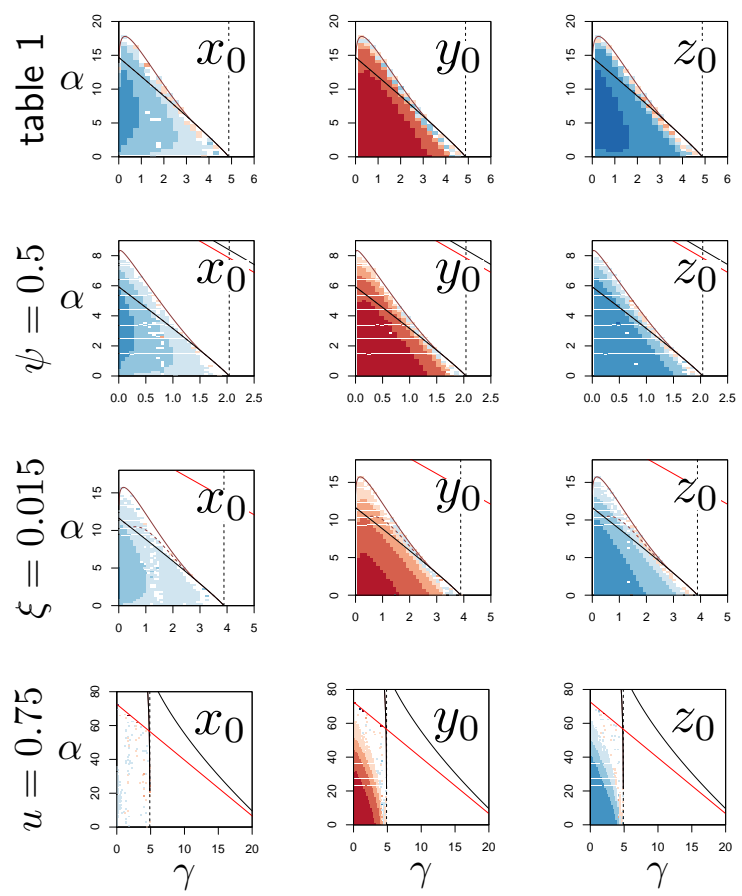

partial derivative of log PLUD relative to log initial conditions

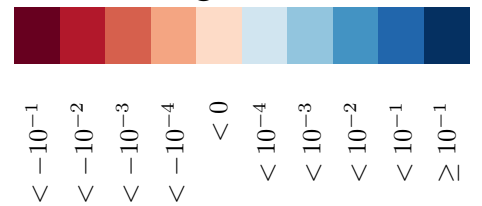

Figure S4-1: Elasticity analysis of the PLUD relative to initial conditions. Each graph represents the partial derivative of $\log$-PLUD relative to $\log$-transformed inoculum size $\left(\log \left(x_{0}\right)\right.$, first column), pre-infection level of defense $\left(\log \left(y_{0}\right)\right.$, second column) and pre-infection level of damage $\left(\log \left(z_{0}\right)\right.$, third column) with $x_{0}=10^{-6}$ and $y_{0}$ and $z_{0}$ set to the homeostatic state. In the first row, parameters are set as in figure 2 , while $\psi=0.5$ in the second row, $\xi=0.015$ in the third and $u=0.75$ in the fourth. The blue color indicates higher PLUD, which could be interpreted as greater tolerance to the infection, while red means lower PLUD. In each case, anything that weakens the host (higher $x_{0}$, lower $y_{0}$ or higher $z_{0}$ ) increases the PLUD which indicates that the PLUD is not an unambiguous measurement of tolerance. In the particular case $u=0.75$, the dependence on $x_{0}$ is so low that numerical derivatives of PLUD cannot be distinguished from zero. 
We shall now demonstrate that this quantity is, in most situations, a lower bound of the PLUD. For this purpose, let us consider the case where $x$ and $z$ both always increase over time, until the host dies. Points on a trajectory leading to host death then necessarily satisfy $H_{x}(x, y)>0$. As $\partial H_{x} / \partial y<0$ and $H_{x}(x, \bar{y}(x))=0$, we can conclude that $y<\bar{y}(x)$ for all points of the trajectory. Because $z$ increases over time, points on the trajectory leading to death must also satisfy $H_{z}(x, y, z)>0$. We know that $\partial H_{z} / \partial x>0$ and $\partial H_{z} / \partial y>0$. Recalling that $y<\bar{y}(x)$, we can therefore conclude that $x>\bar{x}(z)$ for all points on the trajectory. From this we can conclude that the trajectory necessarily reaches $z=z_{d}$ for a value of $x$ which is greater than $\bar{x}\left(z_{d}\right)$ :

$$
x_{d} \geq\left(\xi z_{d}-\eta / \delta\right) /(\omega-\eta / \delta) .
$$

Under some circumstances, though, $x$ and $z$ might decrease as $z$ approaches $z_{d}$. This happens for trajectories which spiral when approaching the high load equilibrium point (i.e. when the Jacobian matrix of the system has two complex eigenvalues for this equilibrium). Numerical simulations indicate, however, that $\bar{x}\left(z_{d}\right)$ is still a lower bound of the PLUD in these conditions.

\section{S4.4 An elasticity analysis of the PLUD}

The PLUD has been proposed as a proxy for tolerance to infection. But the lower bound we obtained in equation (S4-1) demonstrates that it varies with both tolerance and resistance parameters. $\delta$, for example, has an influence on the PLUD lower bound. The more complete elasticity analysis presented in figure S4-2 further demonstrates these mixed influences.

In most cases, the PLUD increases when control of proliferation is loosened (see derivatives according to $\alpha, \beta, \gamma, \psi, \varphi, u, v, k, l$ and $\delta$ in figure S4-2) and when damage production is lowered (see derivatives according to $\omega, \eta$ and $\xi$ in figure S4-2) or the host's tolerance to damage increased (see derivatives according to $z_{d}$ in figure S4-2). Exception to this rule do exist, though. An increase in $\eta$ can indeed either decrease the PLUD, as expected; but under certain parameter combinations it can also increase the PLUD. This happens when both $\alpha$ and $\gamma$ are low in figure S4-2 and probably comes from the fact that increasing $\eta$ strengthens the negative feedback of damage on defense production. 

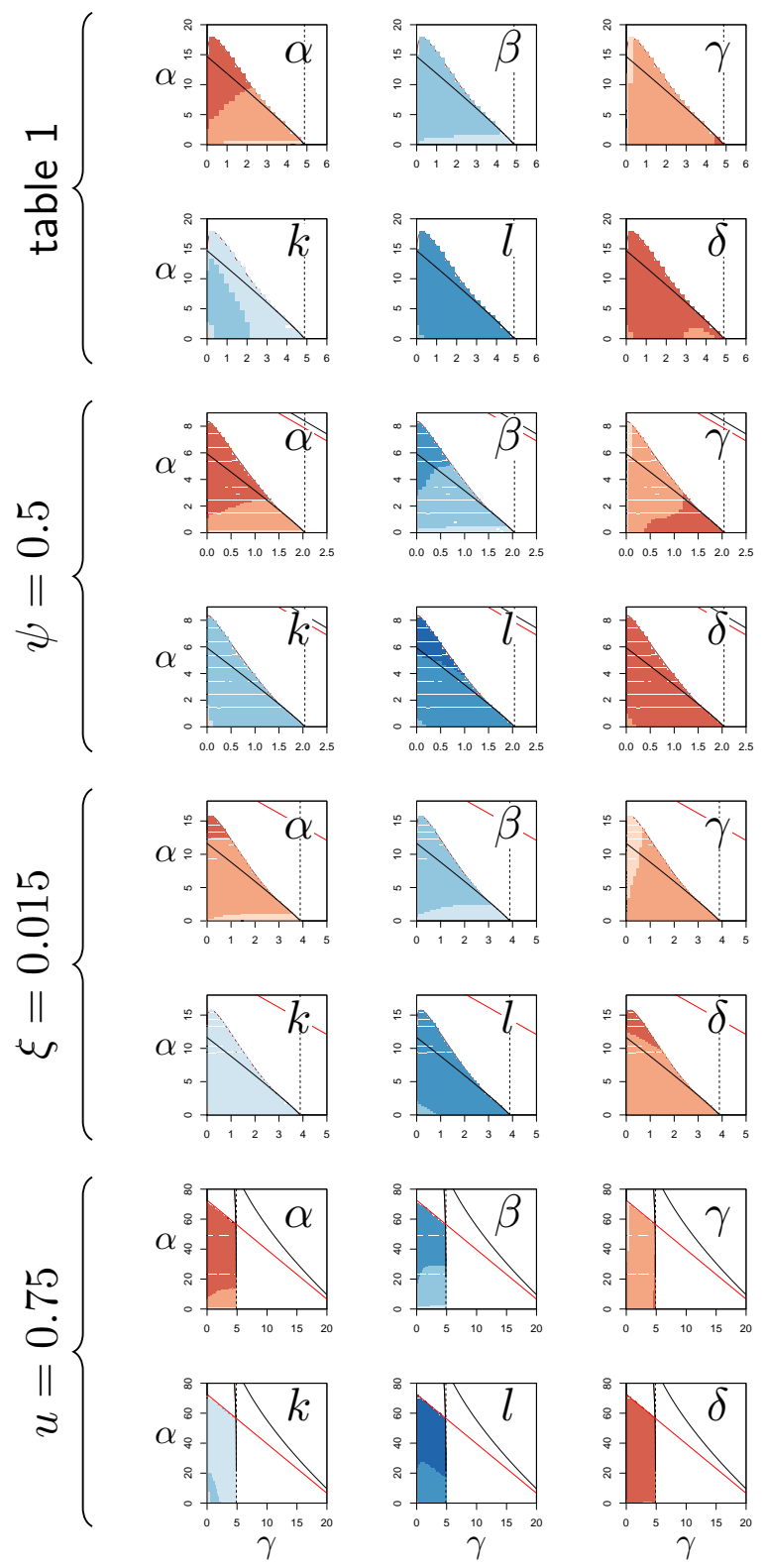

partial derivative of log PLUD relative to log parameters
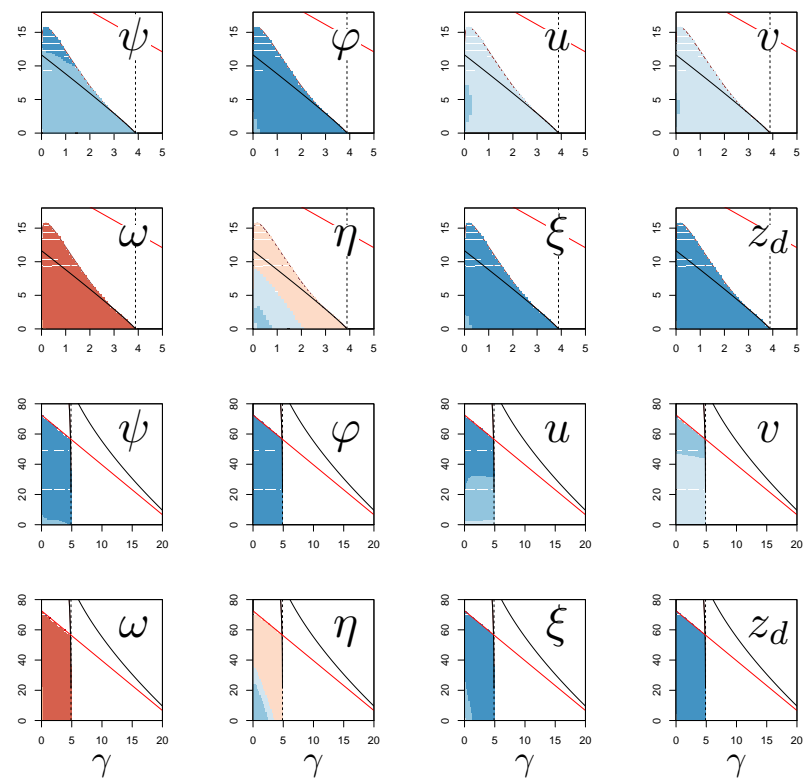

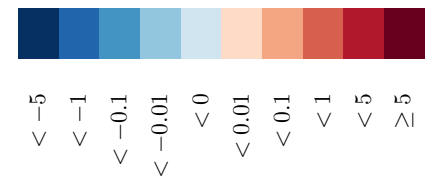

Figure S4-2: Elasticity analysis of PLUD. Each graph represents the partial derivative of PLUD with respect to one parameter, as a function of $\alpha$ and $\gamma$. Blue color corresponds to negative derivatives (i.e. increasing the parameter decreases the PLUD) while red indicate positive derivatives (i.e. increasing the parameter increases the PLUD). The black curves delimit the region of bistability, as in figure 4; above the red line, the equilibrium $z$ is below $z_{d}$ even for the most severe infection. The four braces correspond to the four parameter sets studied in figure 4 . 


\section{S5 Using Hazard Ratio (HR) to compare the lethality of two infections}

\section{S5.1 Definitions}

Mortality measurements are by definition metrics which summarize survival curves. The median and average time to death (or Lethal Time, LT) are among those. One limitation of this type of statistics is that their interpretation might vary depending on the actual distribution of time to death. Another practical limitation is that their computation is problematic for mild pathogens which kill only a small proportion of the infected hosts. An alternative and popular approach is to use Hazard Ratios (HR) to compare one group of hosts (or pathogen) to another group used as a reference. The HR at time $t$ is the ratio of the death probabilities in the focal group to that in the reference group, computed for individuals that have survived up to time $t$. No assumptions are made here on the distribution of time to death, but HR are often assumed to be constant over time (as in the classic Cox Proportional Hazard model). Under this assumption, the comparison of two survival curves therefore yields one unique $\mathrm{HR}$ value.

We chose here to use HR as a convenient way to quantify relative death risk. Simulations proved that our model can produce survival curves which do not necessarily fulfill the proportional hazard hypothesis. We nevertheless found that deviations from this assumption were sufficiently mild to use HR in our analyses.

\section{S5.2 An elasticity analysis of the HR}

We then ran an elasticity analysis of HR relative to both initial conditions and parameter values, following the same logic than for the SPPL and the PLUD. In this analysis, we computed one survival curve for a reference situation and a second one with either one parameter or one initial condition changed. We then compared the two curve using a Cox Proportional Hazard model, which yielded an estimate of HR. The survival curve for the reference group was obtained by simulating infections for 100 individual hosts, with $y_{0}$ set at the homeostatic state. $\log \left(x_{0}\right)$ and $\log \left(z_{0}\right)$ were randomly drawn in Gaussian distributions with averages $\log \left(10^{-6}\right)$ and $\log \left(z_{h}\right)$, respectively, and variance set to 0.25 in both cases (as in the simulations presented in figure $6 \mathrm{C}$ ).

As expected, increasing the dose (increasing $x_{0}$ ), weakening immune defense (lowering $y_{0}$ ), or damaging the host (increasing $z_{0}$ ) upon infection increase HR. We nevertheless found that alterations of $y_{0}$ and $z_{0}$ have much stronger impacts that changes in the dose (see. fig S5-1).

The analysis of how parameter changes influence mortality demonstrates that, as expected, any parameter variation which allows tighter control of proliferation reduces the hazard ratio (see derivatives according to $\alpha, \beta, \gamma, \psi, \varphi, u, v, k, l$ and $\delta$ in figure S5-2). As expected also, parameter variations which reduce the production of damage (see derivatives according to $\omega$, $\eta$ and $\xi$ in figure S5-2) or increase the host's tolerance to damage (see derivatives according to $z_{d}$ in figure S5-2) do reduce the HR. We never observed any exception to this rule. 

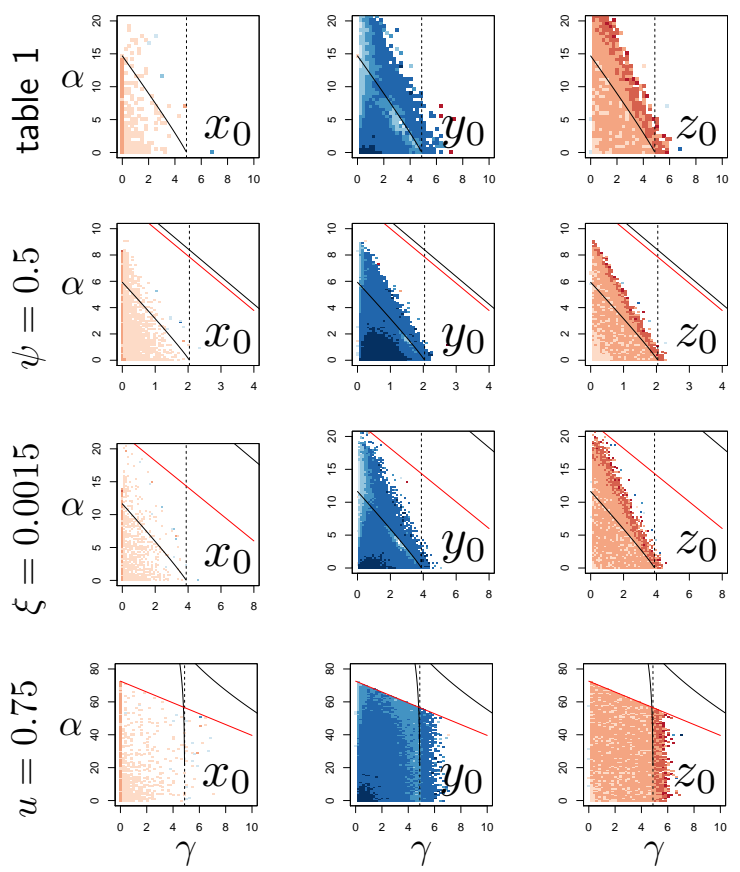

partial derivative of log hazard ratio relative to log initial conditions

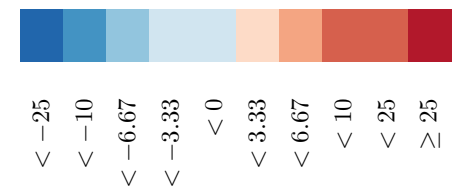

Figure S5-1: Elasticity analysis of Hazard Ratio, relative to initial conditions. Each graph represents the partial derivative of $\log (\mathrm{HR})$ with respect to the log-transformed initial state of one variable, as a function of $\alpha$ and $\gamma$. Blue color corresponds to negative derivatives (i.e. increasing the variable decreases HR) while red indicate positive derivatives. The black curves delimit the region of bistability, as in figure 4; above the red line, the equilibrium $\tilde{z}$ never exceeds $z_{d}$ so that the infection cannot kill the host. Each of the four row corresponds to one of the parameter sets studied in figure 4 . 

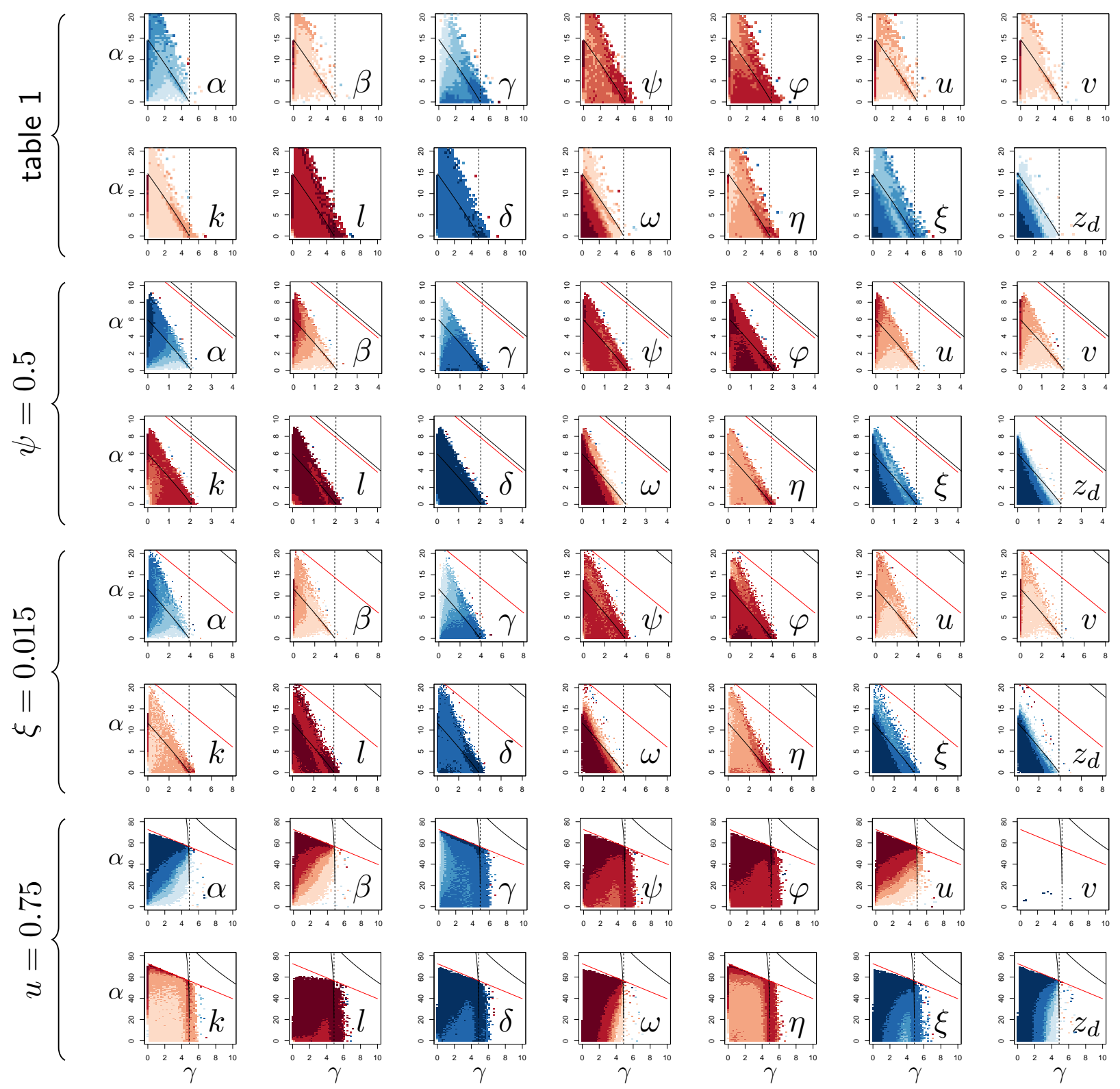

partial derivative of log hazard ratio relative to log parameters

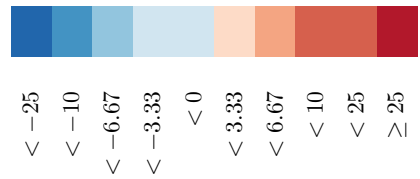

Figure S5-2: Elasticity analysis of Hazard Ratio, relative to parameters. Each graph represents the partial derivative of hazard ratio with respect to one parameter, as a function of $\alpha$ and $\gamma$. Blue color corresponds to negative derivatives (i.e. increasing the variable decreases HR) while red indicate positive derivatives. The black curves delimit the region of bistability, as in figure 4; above the red line, the equilibrium $\tilde{z}$ never exceeds $z_{d}$ so that the infection cannot kill the host. Each of the four rows corresponds to one of the parameter sets studied in figure 4 . 


\section{S6 Measuring the correlation between HR and PLUD: a case test using Drosophila melanogaster}

D. melanogaster were reared until adulthood on glucose-yeast medium $(82 \mathrm{~g} / \mathrm{L}$ yeast, $82 \mathrm{~g} / \mathrm{L}$ glucose, $10 \mathrm{~g} / \mathrm{L}$ agar, propionic acid $3 \mathrm{~g} / \mathrm{L}$, Tegosept $3 \mathrm{~g} / \mathrm{L}$ ) in large fly glass bottle. At day 2 after hatching, adults were isolated in groups of approximately 50 males and 50 females per 900-mL plastic boxes. Husbandry and experiments were conducted at $24^{\circ} \mathrm{C}\left( \pm 1{ }^{\circ} \mathrm{C}\right)$ with a 12 h:12 h light:dark cycle. We used four D. melanogaster lines sampled from the Drosophila Genetic Reference Panel (Mackay et al., 2012, DGRP: RAL-630, RAL-818, RAL-559 and RAL-584) and four genotypes (BOM, A, B and AB, see text for further details) described in (Hanson et al., 2019) which were kindly provided by Bruno Lemaitre's laboratory from the Swiss Federal Institute of Technology in Lausanne.

All experiments were conducted with mated males 5 to 8 days post-eclosion. Males were infected with a strain of Providencia rettgeri isolated from wild-caught D. melanogaster (strain Dmel Galac \& Lazzaro, 2011; Juneja P, 2009) which is moderately virulent (i.e., kills $20-50 \%$ of the individuals over three days when hosts are infected with approximately 1500 bacteria). Liquid cultures of bacterium were grown to saturation overnight at $37^{\circ} \mathrm{C}$. Saturation cultures were pelleted, then resuspended and diluted in phosphate buffered saline (PBS, pH 7.4) to an optical density (OD) of 0.1 (600 nm wavelength). We injected $23 \mathrm{~nL}$ of bacterial suspension into each fly abdomen using a Nanoject II (Drummond). For each experiments, we confirmed that this injection corresponded to an inoculum of approximately 1500 viable bacteria per fly by injecting the suspension in PBS and estimating the number of viable bacteria as described below. Flies were anesthetized with CO2 during the injection procedure, and then were observed shortly after injection to confirm recovery from manipulations. We verified that flies injected with PBS did experience minimal to no mortality during the experiments. After injection, flies were kept together in 900-mL plastic boxes, labelled with information about treatment and genotype, with ad libitum access to food.

\begin{tabular}{|c|c|c|}
\hline Dilution & $\begin{array}{c}\text { Volume }(\mu L) \text { bact. } \\
\text { suspension / PBS }\end{array}$ & $\begin{array}{c}\text { Number of plate } \\
\text { replicates }\end{array}$ \\
\hline \hline 10 & $10 / 90$ & 1 \\
100 & $10 / 90$ & 0 \\
1,000 & $10 / 90$ & 1 \\
5,000 & $40 / 160$ & 2 \\
25,000 & $40 / 160$ & 4 \\
125,000 & $40 / 160$ & 4 \\
625,000 & $40 / 160$ & 4 \\
$1,250,000$ & $50 / 50$ & 2 \\
\hline
\end{tabular}

Table S6-1: Protocol of dilution to estimate the PLUD. 
To monitor PLUD, dead flies were removed every 30min from 12 to 24 hours after injection. Flies which just died were individually homogenized in $250 \mu \mathrm{L}$ of sterile PBS with a TissueLyser homogenizer (Qiagen) with $2 \mathrm{~mm}$ glass beads. The homogenate was diluted in PBS (see table S6-1 for details on the dilution protocol) and a $5 \mu \mathrm{L}$ drop of each dilution was deposited onto LB agar with Erythromycine added $(5 \mu \mathrm{g} / \mathrm{mL}$ final concentration), using a Viaflo multichanel pipette (Integra). Number of replicates for each drops are indicated in table S6-1 and defined to maximise accuracy of the estimation. Plates were incubated overnight at $37^{\circ} \mathrm{C}$ and scanned so that bacterial colonies could be subsequently counted. The number of viable bacteria was estimated for each fly as the intercept of a Poisson GLM with an offset corresponding to the log transformed dilution factor. An average estimate was obtained for each line by adjusting a Negative-Binomial GLM with 'Line' as a main effect and 'Replicate' as random effect using the R package 'spaMM' (Rousset \& Ferdy, 2014). We realised that CFU counts could be under-estimated when density is high and many CFUs overlap. We therefore decided to ignore drops with more than 50 counted CFUs in our analyses. Count of CFU were performed without knowing the information (i.e. genotype or treatment) about the individuals.

We monitored survival first over the same period than the PLUD, and subsequently $24 \mathrm{~h}$ and $48 \mathrm{~h}$ post injection. Individuals alive after 48 days were recorded as censored observations. Host survival differences were analyzed using a Cox's proportional hazards mixed model, with 'Line' as main effect and 'Replicate' as random effect using the R package 'coxme' (Therneau, 2020). 